\title{
Towards Wi-Fi Halow Signal Coverage Modeling in Collapsed Structures
}

\author{
Muhammad Faizan Khan ${ }^{\dagger}$, Guojun Wang ${ }^{* \dagger}$, Md Zakirul Alam Bhuiyan ${ }^{\dagger \dagger}$, and Kun Yang ${ }^{\S}$ \\ ${ }^{\dagger}$ School of Computer Science, Guangzhou University, Guangzhou, China, 510006 \\ ${ }^{\ddagger}$ Department of Computer and Information Sciences, Fordham University, New York, NY, 10458 \\ ${ }^{\S}$ School of Computer Science and Electronic Engineering, University of Essex, Colchester, England \\ ${ }^{*}$ Correspondence to: csgjwang@gzhu.edu.cn
}

\begin{abstract}
With the emerging concept of Wi-Fi radio as sensors, we are witnessing more device-free sensing applications. But we have an observation that most of the existing works of these applications are meant for simple indoor layout and are not adequate for complex cases, e.g., collapsed structures. In this paper, we explore the feasibility of Wi-Fi Halow signals for the collapsed scenario as it can boost rescue efforts. To achieve this, we aim at two prime objectives of this work. Firstly, we model debris constituent of common collapsed scenario materials such as concrete, brick, glass, and lumber by conducting a field survey of an earthquake affected area. After that, we consider signal propagation models for better coverage in this debris model by employing two methods. The first method is an integrated TOPSIS and Shannon Entropy-based on bijective soft set which provides us an approximation tool to select the best Wi-Fi Halow signal coverage in debris. The second method composes two modified wireless signal propagation models, which are transmitter-receiver (TR) and Wi-Fi radar, respectively. We perform extensive simulations and figure out that low power transmission using Wi-Fi radar can yield better coverage, which is also verified by the Shannon entropy method.
\end{abstract}

Index Terms-Wi-Fi Halow, IoT, coverage, collapsed structure, soft set, rescue, path loss.

\section{INTRODUCTION}

$\mathbf{W}$ ITH the realization of leveraging Wi-Fi radio as sensors, the research community is paying more attention towards device-free sensing applications [1], as these are being considered the future of IoTs, where widget free sensing will be more imminent. But, there are certain areas such as human rescue from collapsed structures, where wireless signal penetration is very weak. So, there is dire need to investigate low-powered wireless signals that may also provide sensing for possible human detection under debris. This is entirely in line with the concept of IoT deployment by paving the way for ubiquitous disaster management. In this paper, we consider Wi-Fi Halow, which is a low-powered Wi-Fi standard primarily designed for IoTs to address the weak signal coverage issue in collapsed structures.

\section{A. Prior Work}

Wireless signal coverage in the complex indoor layout has been extensively studied within the domain of indoor wireless communication. We observe through studies $[2,3]$ that attenuations, fading, higher path losses, and scattering, etc. adversely affect the wireless signals in indoor environments.
And it becomes even worse for the case of a collapsed indoor structure; where roofs, walls, partitions, and doors turn into multilayered debris. So, wireless signal penetration through debris seems almost impossible. But, we do need to study the wireless signals for these collapsed structures as it may lead to the post-disaster rescue.

It has been observed that structural collapses are widespread everywhere in the world, which, unfortunately, results in the loss of precious human lives. Although, there are some techniques available in the literature to boost the rescue efforts which have been manual by large till today. These solutions are based on cameras [4, 5], robots [6], sensors [7], and radars [8]. We have an observation that the most promising one is the radar-based technique among all others which recognize the micro-signatures [9] and phase change from echo at low frequency $[10,11]$. But these techniques are by large susceptible to noises and also very much expensive which limits their applications as most of the developing nations cannot utilize these methods, where major collapses occur due to poor infrastructure and low engineering standards. So, there is dire need to find an alternative solution which should be both ubiquitous and cost-effective.

Recently, Wi-Fi radios are being leveraged as sensors for various indoor IoT applications. These signals have sensing capability because of channel state information (CSI) [12] that encompasses this information of the desired event from the surroundings within the channel. Some of the most prominent Wi-Fi signal applications are; WiGest [13], CSI-based indoor localization [14], WiFi-ID [15], WiHear [16], Mo-sleep [17], breathing detection [18], WiKey [19], WiDraw [20], RTFall [21], security [12], and privacy [22] to name a few. We observe that indoor localization and breathing detection employing Wi-Fi signals can make a turn-around in rescue efforts if these can be employed in the complex indoor layout such as collapsed structure. We envision that there is a dire need to investigate the Wi-Fi signal penetration and to increase the signal strength under debris because it may lead to device free post-disaster rescue which will be quite cost-effective and ubiquitous as well. Based on this motivation, we focus our research on the coverage aspect of low-frequency Wi-Fi signals that may open new dimensions in rescue solutions.

There are two fundamental challenges to $\mathrm{Wi}-\mathrm{Fi}$ signals for adequate coverage in collapsed structures, which are path loss models and signal operating frequencies. We observe 
that most of the existing radar sensing techniques operate at low frequencies as high-frequency signals face more attenuations, improper reflections, fading, and scattering from debris. There are some research studies like [23]-[25] dealing various frequencies ranging from $50 \mathrm{MHz}$ to $2.4 \mathrm{GHz}$ for complex structures, which convince us that low-frequency wireless signals can penetrate better in collapsed structures. But none of these works explicitly dealt with Wi-Fi radios as sensors. Similarly, path loss modeling has been widely studied such as [2, 3, 26]-[29], but unfortunately, these works do not analyze the path losses for collapsed structure case. To the best of our knowledge, all the existing studies related to path loss models consider source and destination at two different locations, which limit coverage of wireless signals in debris as damping, attenuations, and fading come into effect. So, there is a dire need to study new methods for wireless path losses in collapsed structures. Recently, there is work by authors in [30]-[33] where they tried to investigate the subject matter and suggested that Wi-Fi Halow [34], which is low power and low-frequency standard from Wi-Fi Alliance, is best suited for adequate coverage in collapsed structures. The current study is an extension of ongoing research by having a more in-depth analysis of path losses with a proper comparison based on strong theoretical and conceptual methodolgy. We also have an observation that research on structural health monitoring like [35]-[37] can provide a breakthrough on coverage computation of wireless signals in complex environments.

\section{B. Contribution and Organization}

The prime objective of this work is to study the Wi-Fi Halow signal coverage in a collapsed structure. We consider two main goals of this study to address coverage adequately. Firstly, we need to develop a debris structural model which should be based on a real field survey to provide a sound basis. To achieve this, we conducted a field survey of an earthquake area of China that resulted in a loss of more than 68 thousand people. We studied the causes of structural collapses, kind of structural materials and impact of the earthquake and developed a layered debris model constituent of concrete, brick, glass, and lumber to map ruins into theoretical replicas. After that, we define the problem through echo modeling based on Doppler radar principle. To address the coverage of Wi-Fi Halow from echo and debris model, we consider two methodologies; which are Shannon entropy TOPSIS based on bijective soft set [38] from the theoretical aspect and coverage range models from wireless signal propagation theory. Shannon entropy TOPSIS method provides a mathematical proof on conceptual understandings of wireless signals in collapsed structures, whereas coverage models properly employ two modified path loss methods for signal losses and attenuations. These methods are transmitter-receiver(TR) and Wi-Fi radar, respectively. Finally, we compare the results from both radio models with Shannon entropy TOPSIS method to figure out the best possible concept for adequate coverage of $\mathrm{Wi}-\mathrm{Fi}$ Halow signal in collapsed structures.

The major contributions of this study are;

- We explore the feasibility of Wi-Fi Halow signals for adequate coverage in the complex indoor layout such as collapsed structures. There can be multiple causes of structural collapses such as earthquakes, tsunamis, and terrorism, etc. So, to address the nature of the debris, we conducted a field survey of an earthquake-affected area as soil mechanics, structural materials, environment, and area economics matters a lot.

- We define our coverage problem through Wi-Fi Halow signal and echo modeling based on the debris model developed from a real field survey. These models provide an in-depth investigation of structural materials and signal propagation mechanism. We envision that wireless signals will be reflected after striking with objects quite similar to Doppler radar method.

- We formulate two methodologies to address the Wi-Fi Halow signal coverage from debris and echo models. Firstly, we present Shannon entropy TOPSIS based on a bijective soft set technique to visualize the wireless signal propagation in debris conceptually. It is based on the selection of proper attributes to provide better coverage using preferential values from radio specialists. After that, we present two coverage range methods, which are TR and Wi-Fi radar, respectively. These both methods have been modified from existing path loss techniques and radar range equation to address the coverage in collapsed structures.

- Finally, we validate our signal propagation models through proper simulations while considering signal intensities, threshold, modulation schemes, debris layers, and Wi-Fi Halow frequency for two countries, i.e., China and U.S.A. Furthermore, we compare these results with conceptual results obtained from Shannon entropy TOPSIS method. We conclude that Wi-Fi Halow signals with low power and the low frequency with a smaller modulation scheme at minimum layered debris yield better coverage results. This inference completely justifies the basic concept of Wi-Fi Halow, which was primarily designed for IoTs, and our results also suggest that low power Wi-Fi Halow signals can probably provide us better coverage. So, we envision to expect IoT based postdisaster rescue solutions around the world in the future.

The remainder of this paper is organized as follows. We discuss problem formulation in Section II, whereas Section III provides the concept for Wi-Fi Halow signal coverage. We present two methodologies i.e, Shannon entropy TOPSIS and coverage range methods in Sections IV and V. Simulations results have been provided in Section VI. After that, we briefly analyze both methods in Section VII along-with contributions and limitations in comparison with existing literature. Finally, Section VIII concludes the paper with future directions.

\section{Problem Formulation}

This section provides the foundation of our study. Firstly, we provide a brief analysis for nature of the collapsed structure through a field survey of an earthquake affected area of southwestern China. Then, we investigate the core reasons along with a study of structural materials. Furthermore, we transform the field survey into a debris model and discuss 


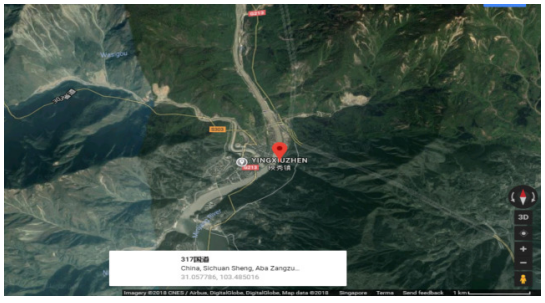

(a) Location of Yingxiu town

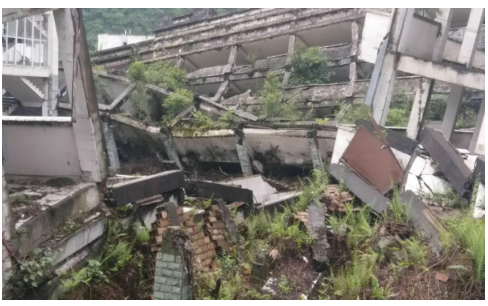

(b) Xuankou middle school

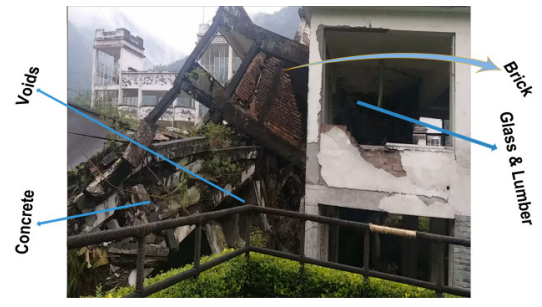

(c) Collapsed materials

Fig. 1: Earthquake survey site: Yingxiu town, Wenchuan County, P.R. China

Wi-Fi Halow signal behavior in this multi-obstructed scenario. Finally, we come up with the echo model, which provides a proper problem formulation.

\section{A. Collapsed Structure Measurement}

The first goal of this study is to find a possible collapsed structure because it may lead to proper debris modeling that can further assist in coverage computation. We observed there are multiple causes of collapsed environments such as earthquakes, tsunamis, terrorist activities, fire incidents, or structural failures. But in this research, we have considered an earthquake as a leading source of structural collapses as the majority of the active fault lines exists mostly in developing countries, where construction standards are not followed adequately due to the weak economy. To cope with this, we conducted a field survey of an earthquake hit area of Sichuan, which is the southwestern province of China. The main reason to select this site was the construction standards as rural areas did not fully comply with building codes, whereas urban areas have been planned according to standards.

The epic center of the earthquake was Yingxiu ${ }^{1}$, a small town in southern Wenchuan county within Sichuan province. It is located at latitude and longitude of $31^{\circ} 03^{\prime} 32^{\prime \prime} \mathrm{N}$ and $103^{\circ} 29^{\prime} 41^{\prime \prime} \mathrm{E}$ respectively, as shown in Fig 1a. Most of the adjoining rural areas of Wenchuan were destroyed by a devastating earthquake that hit China with a moment magnitude of 7.9 on May $12,2008^{2}$. Government sources put the casualties' number to more than 68 thousand. We conducted an indepth investigation from the ruins of Xuankou Middle school, as shown in Fig 1b located in the town of Yingxiu. We observed that structural collapse was caused by poor building construction with concrete, brick, glass, and lumber, as shown in Fig 1c. This collapse also caused voids, where there was a possibility of humans. Therefore, a proper study containing reasons for structural failure can lead to the better rescue. Our analysis showed that the columns were not reliable, and also the steel binding in the core basement did not meet construction codes. Even the beam was not up to the mark and collapsed with the first shock. In short, construction materials with poor standard were the primary source of collapse. So, in this study, we focus our research on Wi-Fi Halow signal penetration through these construction materials that are commonly used in all developing countries.

\footnotetext{
${ }^{1}$ https://en.wikipedia.org/wiki/Yingxiu

${ }^{2}$ https://earthquake.usgs.gov/earthquakes/eventpage/usp000g650\#executive
}

\section{B. Debris Model}

Let us map our field survey from Fig 1c to theory through a debris model. It is an established fact that the selection of adequate debris is pivotal for coverage computation in collapsed structures. This is evident from the debris that structures after collapse turn up into multiple objects laying over each other. Furthermore, there can be various materials in this debris as well, but we have only considered brick, concrete, glass, and lumber to address both structural complexities and to simplify the problem as shown in Fig 2a.

Let us dive into further depth of this debris model. The geometry of debris is also a significant factor which classifies it into horizontal and vertical directions. We consider it as layered debris where multiple objects laying over each other defines the depth and width of debris. The extent of debris is considered through vertical layers, and our layered model primarily focuses on this. We further classify these vertical layers into minimum and maximum layered model, as seen in Fig $2 \mathrm{~b}$ and Fig $2 \mathrm{c}$. The reason for this classification is to figure out the maximum possible coverage of Wi-Fi Halow signal. It is quite evident that the signal will damp after penetration through various materials, but we need to find the maximum depth that can provide us some coverage.

\section{Wi-Fi Halow Echo Model}

Let us consider the Wi-Fi Halow signal behavior in the above-developed debris model, as shown in Fig 2a. Collapsed structures are far complex than typical indoor scenarios primarily because of the multilayered debris formed from halls, walls, and door, etc. So, it becomes very much difficult for the wireless signal to penetrate and reflect from debris. Most of the times, there is a chance of signal damping because of various fading phenomena's. But, there is still a need for in-depth investigation for $\mathrm{Wi}-\mathrm{Fi}$ signals as it may turn into post-disaster rescue solution.

To simplify the coverage computation, we consider a WiFi Halow echo model, as shown in Fig 2d. We assume that wireless signals follow the Doppler radar principle. There is a stream of wireless pulses penetrating debris which will either be reflected or damped. Our goal is to get a proper echo which may provide us suitable CSI. Although, we may get signal coverage with very low sensitivity i-e., $-100 \mathrm{dBm}$, but that reflections will be very weak and we cannot extract CSI from the received signal. So, there is a need for a strong echo signal. To achieve this, echo signals should be above than the predefined threshold (PDT). We only consider signals from 


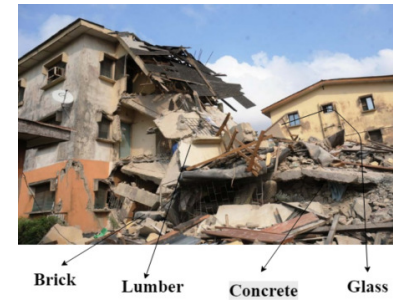

(a) Collapsed Model

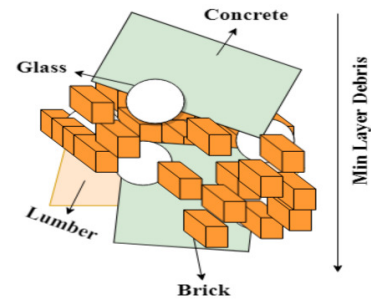

(b) Minimum layers

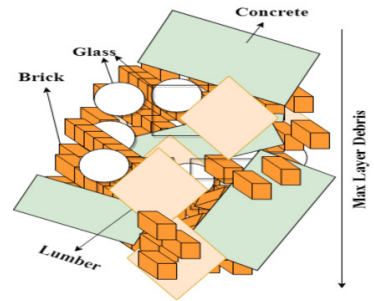

(c) Maximum layers

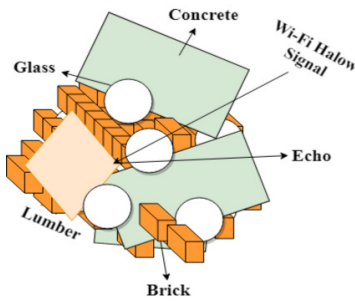

(d) Wi-Fi Halow echo

Fig. 2: Earthquake survey site with echo and layered models

reflected from debris while other signals are assumed to be noise.

T $\mathrm{N}$ a nutshell, our main objective is to have better coverage for Wi-Fi Halow signal from collapsed structures as these face higher attenuation in debris. So, there is a need to formulate methods based on echo and debris models that may assist in comprehending and computing the Wi-Fi signals.

\section{Wi-Fi Halow Coverage ConcePt}

This section provides insight into the coverage concept of Wi-Fi Halow signals for collapsed structures. Firstly, the significance of Wi-Fi Halow signal coverage is discussed which draws our attention towards low-frequency study in debris scenarios. After that, we concisely explain the adopted methodologies to ensure better coverage.

The prime objective of this research is to study the indepth feasibility of Wi-Fi Halow signals for debris scenarios as [31] states that low frequency is the optimal solution for coverage in collapsed structures. Low frequencies result in higher wavelength, which ensures better diffraction of the beam. It can also excite delocalized electrons in a metal that allows penetration of low-frequency signal better than higher ones. It is also evident from [39] that Wi-Fi Halow has been designed primarily for IoTs. This concept perfectly justifies our idea of low power, ubiquitous post-disaster rescue solution. Therefore, we focus on the coverage aspect of Wi-Fi Halow signals, which may turn into a rescue solution soon in the future.

Let us discuss the methodologies that may provide us understandings of coverage concept. We consider two techniques, as shown in Fig 3a, where Shannon entropy TOPSIS based on bijective soft set is employed from operational research [38] to ensure better decision making at the abstract level. After that, coverage range methods from the wireless domain provides insight from radio propagation perspective. We consider two approaches i-e., simple transmitter-receiver mechanism, and radar technique to map the attenuations and losses in debris. The difference between both methods is the placement of receiver, which in TR-approach is at a separate place than the transmitter, whereas in Wi-Fi radar technique, its same device. Finally, we make a comparison between both methods and figure out the best coverage concept from both simulation and theoretical aspect.

\section{SHANNON ENTROPY TOPSIS}

In this section, we employ a conceptual model using Shannon entropy TOPSIS based on bijective soft set to select the best Wi-Fi signal attributes for coverage in collapsed structures. But, before going into the detail of the conceptual model, we firstly discuss the motivation behind employing this technique from operational research to computer science. After that, we provide some preliminary definitions followed by the method and its operation to the debris model.

We know that decision making from multi-criteria attributes is one the biggest challenge in operational research, and researchers have been proposing new methods to have effective decisions. A significant contribution was soft sets which were introduced by Molodtsov [40], and followed by a study [41], that proposed bijective soft set. Furthermore, [42] extended soft sets to intuitionistic fuzzy soft sets. These studies suggest that soft sets are handy for decision making and to deal with uncertainties. Now, our problem also needs some conceptual level decision making before specifically proposing wireless propagation models, because Wi-Fi signal penetration in collapsed structures is entirely uncertain. We cannot randomly decide some wireless propagation methods. There is a need to consult the literature and radio specialists to realize the aforediscussed field survey in the context of Wi-Fi Halow signals. Therefore, we need to conceptually analyze the problem and make trustworthy decisions which may help us to employ path loss models effectively. Motivated by study [38], which is based on Shannon entropy weights, we apply this approach for Wi-Fi Halow signals as it covers both wireless aspect and theoretical bijective soft set as shown in Fig $3 b$.

\section{A. Preliminary Definitions}

Let us consider the fundamental operation and definition of soft sets as it would make subsequent sections more understandable.

1) Soft Set: Suppose we have set of parameters and universal set as $S$ and $U$ respectively. Let $P(U)$ be the power set of $\mathrm{U}$, and $\mathrm{X}$ is a subset of $\mathrm{S}$ as $\mathrm{X} \subset \mathrm{S}$. Then, a pair $(\mathrm{F}, \mathrm{X})$ is known as a soft set over $\mathrm{U}$, where mapping function $\mathrm{F}$ is given by $\mathrm{F}: \mathrm{X} \rightarrow \mathrm{P}(\mathrm{U})$ [43].

2) Bijective Soft Set: Let (F, S) be a soft set over universal set $\mathrm{U}$ and $\mathrm{S}$ as a non-empty set of parameters. Then, (F, S) is known as bijective soft set [41] if the following conditions are met:

i $\bigcup_{\beta \in S} F(\beta)=U$.

ii For any two parameters; $\beta_{i}, \beta_{j}, \beta_{j} \in S, \beta_{i}=\beta_{j}, \mathrm{~F}\left(\beta_{i}\right) \cap \mathrm{F}\left(\beta_{j}\right)=\varnothing$. 
1

2

3

4

5

6

7

8

9

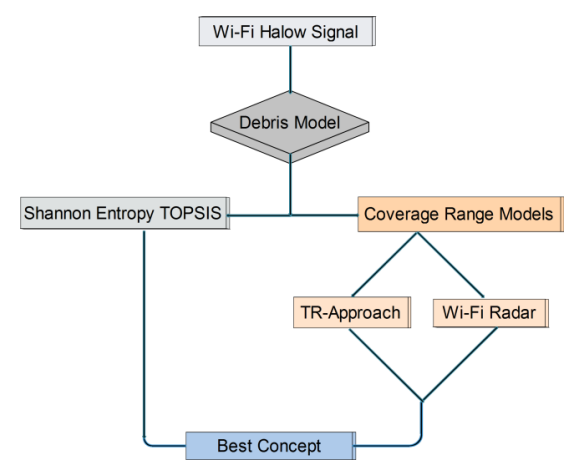

(a) Coverage concept

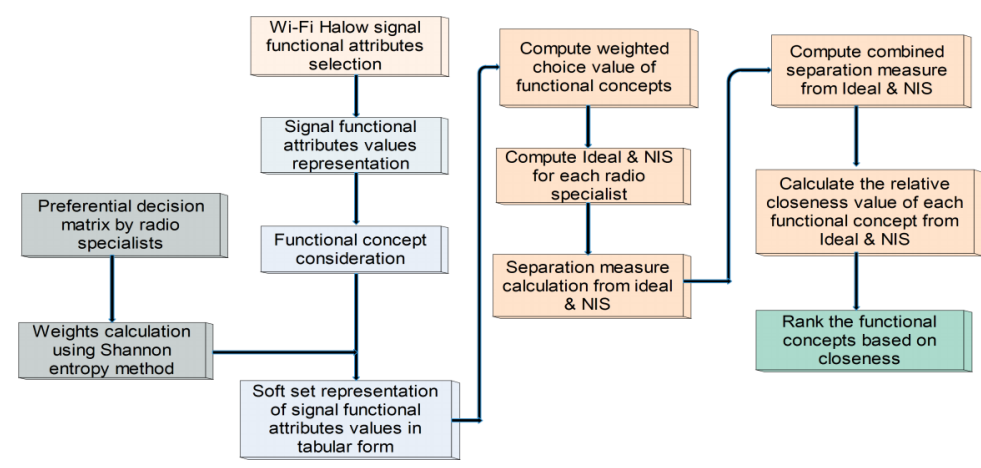

(b) Proposed functional concept methodology

Fig. 3: Illustration of coverage concepts

\section{B. Method}

Input: Set of Wi-Fi Halow signal functional requirements Output: Best Wi-Fi Halow coverage concept

1) Identify the Wi-Fi Halow signal functional attributes which matters the most for debris scenario. After that, assign possible conceptual values to these attributes by following standard communication standards and represent it in a set form.

2) Generate functional concepts for the signal from attribute values that are most suited for coverage in collapsed structures and discard others. These concepts provide a theoretical understanding of $\mathrm{Wi}-\mathrm{Fi}$ signal penetration in debris.

3) Represent attributes values in both soft set and bijective soft set form to proceed for decision making.

4) Capture the Wi-Fi Halow signal preferences for radio specialists on the abstract level and form preferential decision matrix as $\mathcal{P} D M=\left[p_{i j}\right]_{a \times b}$, where $i=1, \ldots, a$ and $j=1, \ldots b ; p_{i j}$ imply the preferences values. Here 'a' and ' $b$ ' represent number of radio specialists and total signal attribute values, respectively.

5) Calculate the projection value $(p v)$, entropy ( $\left.\mathbb{E}_{n t}\right)$, divergence $(\mathfrak{D} i v)$, and weight $(\mathfrak{W} g t)$ of each signal attribute value $\gamma_{i j}$ from [44] as below;

$p v_{i j}=\frac{p_{i j}}{\sum_{i=1}^{a} p_{i j}}$, Ent $=-\kappa \sum_{i=1}^{a} p v_{i j} \ln \left(p v_{i j}\right)$

where $\kappa$ is a constant implied as, $\kappa=(\ln (a))^{-1}$, then $\operatorname{Div}=1-(\mathfrak{E} n t), \mathfrak{B} g t\left(\gamma_{i j}\right)=\sum_{\kappa=1}^{n} \frac{\mathfrak{D} i v_{j}}{\mathfrak{D} i v_{\kappa}}$

6) Capture the requirements from radio specialists $\mathcal{R} S R$ that may provide better coverage for Wi-Fi Halow signal.

7) Represent the Shannon entropy weights in soft set tabular form and compute weighted choice value $\mathcal{W} C V$ for each radio preference scheme through respective attributes and functional concepts as; $\mathcal{W} C V_{i \kappa}=\sum_{j} \mathfrak{D} i v_{i j}$, where $\mathfrak{D} i v_{i j}=$ $\mathfrak{W} g t\left(\gamma_{i j}\right) \times q_{i j}$. Here $q_{i j}$ is obtained from signal functional concepts.

8) Determine ideal (IS) and Non-ideal solution (NIS) as $\gamma_{i}^{*}$ and $\gamma_{i}$ for each radio specialist using TOPSIS as below; $\gamma_{i}^{*}=\mathcal{M} \operatorname{lax}\left(\mathcal{W} C V_{i K}\right) ; \gamma \gamma_{i}=\mathcal{M} i n\left(\mathcal{W} C V_{i K}\right)$

9) Calculate the separation measure $\left(\Delta_{i \kappa}^{*}, \Delta_{i \kappa}\right)$ of each functional concept from the IS and NIS using n-dimensional Euclidean distance for each radio specialist by following relation; $\Delta_{i K}^{*}=\left(\gamma_{i j}-\gamma_{i}^{*}\right)^{2}, \breve{\Delta_{i K}}=\left(\gamma_{i j}-\breve{\gamma_{i}}\right)^{2}$
Then combined separation measure $\left(\Delta_{K}^{*}, \breve{\Delta_{K}}\right)$ for each functional concept will be computed as below;

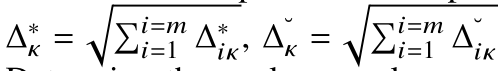

10) Determine the analogous closeness of each signal functional concept $\mathcal{F} \zeta_{K}$ to IS, which is provided as; $\zeta_{K}^{*}=$ $\frac{\Delta_{\kappa}^{\check{2}}}{\overline{\Delta_{\kappa}+\Delta_{\kappa}^{*}}}$

The most closer measure to the concept will be the best coverage of Wi-Fi Halow signals in collapsed structures.

\section{Operation}

We can apply the Shannon entropy TOPSIS method based on bijective soft set to our Wi-Fi Halow coverage problem as;

i Wi-Fi Halow coverage requirements can be defined by five signal functional attributes(SFAs) to form a SFA set as per 1: $\mathcal{S F \mathcal { A }}=\left[\mathcal{S} \mathcal{F} \mathcal{A}_{1}, \mathcal{S} \mathcal{F} \mathcal{A}_{2}, \mathcal{S} \mathcal{F} \mathcal{A}_{3}, \mathcal{S} \mathcal{F} \mathcal{A}_{4}, \mathcal{S F} \mathcal{A}_{5}\right]$, where these attribtes represent following;

$\mathcal{S F} \mathcal{A}_{1}=$ Power, $\mathcal{S} \mathcal{F} \mathcal{A}_{2}=\mathcal{M} D S$ threshold, $\mathcal{S} \mathcal{F} \mathcal{A}_{3}=$ Coverage, $\mathcal{S} \mathcal{F} \mathcal{A}_{4}=$ Echo reception probability, $\mathcal{S F} \mathcal{A}_{5}=$ Debris layers

Furthermore, there is a need to assign values to these $\mathcal{S F} \mathcal{A}$ s according to standard regulatory bodies. We denote these values with $\gamma$ as follows;

$\mathcal{S F} \mathcal{A}_{1}=\left\{\gamma_{11}, \gamma_{12}, \gamma_{13}\right\}=\{$ High, Medium, Low $\}$

$\mathcal{S F} \mathcal{A}_{2}=\left\{\gamma_{21}, \gamma_{22}, \gamma_{23}\right\}=\{$ V. Good, Good, Poor $\}$

$\mathcal{S F} \mathcal{A}_{3}=\left\{\gamma_{31}, \gamma_{32}, \gamma_{23}\right\}=\{$ V. Good, Acceptable, Low $\}$

$\mathcal{S F} \mathcal{A}_{4}=\left\{\gamma_{41}, \gamma_{42}, \gamma_{43}\right\}=\{$ V. Good, Acceptable, Low $\}$

$\mathcal{S F} \mathcal{A}_{5}=\left\{\gamma_{51}, \gamma_{52}\right\}=\{$ Maximum, Minimum $\}$

ii We generate five Wi-Fi Halow functional concepts as per 2 by forming an appropriate combination from $\mathcal{S} \mathcal{F} \mathcal{A s}$ which can be provided in a universal set given as;

$\bigcup=\mathcal{F} \zeta_{1}+\mathcal{F} \zeta_{2}+\mathcal{F} \zeta_{3}+\mathcal{F} \zeta_{4}+\mathcal{F} \zeta_{5}$

We present these functional concepts as sets below;

$\mathcal{F} \zeta_{1}=\left\{\gamma_{11}, \gamma_{21}, \gamma_{33}, \gamma_{43}, \gamma_{52}\right\}$

$\mathcal{F} \zeta_{2}=\left\{\gamma_{11}, \gamma_{23}, \gamma_{33}, \gamma_{43}, \gamma_{52}\right\}$

$\mathcal{F} \zeta_{3}=\left\{\gamma_{12}, \gamma_{22}, \gamma_{32}, \gamma_{43}, \gamma_{52}\right\}$

$\mathcal{F} \zeta_{4}=\left\{\gamma_{13}, \gamma_{21}, \gamma_{32}, \gamma_{42}, \gamma_{51}\right\}$

$\mathcal{F} \zeta_{5}=\left\{\gamma_{13}, \gamma_{21}, \gamma_{31}, \gamma_{41}, \gamma_{51}\right\}$

iii Soft sets which may provide us an indirect representation of desired signal specification from $\mathcal{F} \zeta$ can be as follows; $\left(\mathcal{S} S_{1}, \mathcal{S} \mathcal{F} \mathcal{A}_{1}\right)=\left\{\mathcal{S} S_{1}\left(\gamma_{11}\right), \mathcal{S} S_{1}\left(\gamma_{12}\right), \mathcal{S} S_{1}\left(\gamma_{13}\right)\right\}$ $\left(\mathcal{S} S_{2}, \mathcal{S F} \mathcal{A}_{2}\right)=\left\{\mathcal{S} S_{2}\left(\gamma_{21}\right), \mathcal{S} S_{2}\left(\gamma_{22}\right), \mathcal{S} S_{2}\left(\gamma_{23}\right)\right\}$ $\left(\mathcal{S} S_{3}, \mathcal{S F} \mathcal{A}_{3}\right)=\left\{\mathcal{S} S_{3}\left(\gamma_{31}\right), \mathcal{S} S_{3}\left(\gamma_{32}\right), \mathcal{S} S_{3}\left(\gamma_{33}\right)\right\}$ 
TABLE I: Preferential Decision Matrix

\begin{tabular}{lllllllllllllll}
\hline & $\gamma_{11}$ & $\gamma_{12}$ & $\gamma_{13}$ & $\gamma_{21}$ & $\gamma_{22}$ & $\gamma_{23}$ & $\gamma_{31}$ & $\gamma_{32}$ & $\gamma_{33}$ & $\gamma_{41}$ & $\gamma_{42}$ & $\gamma_{43}$ & $\gamma_{51}$ & $\gamma_{52}$ \\
\hline $\mathfrak{R} S_{1}$ & 0.1 & 0.4 & 0.8 & 0.6 & 0.4 & 0.1 & 0.8 & 0.6 & 0.1 & 0.8 & 0.6 & 0.4 & 0.8 & 0.4 \\
$\Re S_{2}$ & 0.4 & 0.8 & 0.6 & 0.4 & 0.6 & 0.8 & 0.6 & 0.4 & 0.4 & 0.4 & 0.4 & 0.1 & 0.4 & 0.6 \\
$\Re S_{3}$ & 0.8 & 0.6 & 0.1 & 0.1 & 0.8 & 0.6 & 0.4 & 0.4 & 0.1 & 0.6 & 0.4 & 0.1 & 0.1 & 0.8
\end{tabular}

TABLE II: Projection, Entropy, Divergence, Weight

\begin{tabular}{|c|c|c|c|c|c|c|c|c|c|c|c|c|c|c|}
\hline & $\gamma_{11}$ & $\gamma_{12}$ & $\gamma_{13}$ & $\gamma_{21}$ & $\gamma_{22}$ & $\gamma_{23}$ & $\gamma_{31}$ & $\gamma_{32}$ & $\gamma_{33}$ & $\gamma_{41}$ & $\gamma_{42}$ & $\gamma_{43}$ & $\gamma_{51}$ & $\gamma_{52}$ \\
\hline $\mathcal{P R S} S_{1}$ & 0.0769 & 0.2222 & 0.5333 & 0.5455 & 0.2222 & 0.0667 & 0.4444 & 0.4286 & 0.1667 & 0.4444 & 0.4286 & 0.6667 & 0.6154 & 0.2222 \\
\hline $\mathcal{P} R S_{2}$ & 0.3077 & 0.4444 & 0.4 & 0.3636 & 0.3333 & 0.5333 & 0.3333 & 0.2857 & 0.6667 & 0.2222 & 0.2857 & 0.1667 & 0.3077 & 0.3333 \\
\hline $\mathcal{P} \mathfrak{R} S_{3}$ & 0.6154 & 0.3333 & 0.0667 & 0.0909 & 0.4444 & 0.4 & 0.2222 & 0.2857 & 0.1667 & 0.3333 & 0.2857 & 0.1667 & 0.0769 & 0.4444 \\
\hline Ent & 0.7817 & 0.9656 & 0.8031 & 0.8342 & 0.9656 & 0.8031 & 0.9656 & 0.9821 & 0.7897 & 0.9656 & 0.9821 & 0.7897 & 0.7817 & 0.9656 \\
\hline Div & 0.2183 & 0.0344 & 0.1969 & 0.1658 & 0.0344 & 0.1969 & 0.0344 & 0.0179 & 0.2103 & 0.0344 & 0.0179 & 0.2103 & 0.2183 & 0.0344 \\
\hline $\mathfrak{B} g t$ & 0.1344 & 0.0213 & 0.1212 & 0.1021 & 0.0213 & 0.1212 & 0.0213 & 0.0110 & 0.1295 & 0.0213 & 0.0110 & 0.1295 & 0.1344 & 0.0213 \\
\hline
\end{tabular}

TABLE III: Soft set representation of $R S_{1}$

\begin{tabular}{ccccccc}
\hline & $\gamma_{13}$ & $\gamma_{21}$ & $\gamma_{31}$ & $\gamma_{41}$ & $\gamma_{51}$ & $\mathcal{W} C V$ \\
\hline $\mathcal{F} \zeta_{1}$ & 0 & 1 & 0 & 0 & 0 & 0.1021 \\
$\mathcal{F} \zeta_{2}$ & 0 & 0 & 0 & 0 & 0 & 0 \\
$\mathcal{F} \zeta_{3}$ & 0 & 0 & 0 & 0 & 0 & 0 \\
$\mathcal{F} \zeta_{4}$ & 1 & 1 & 0 & 0 & 1 & 0.3577 \\
$\mathcal{F} \zeta_{5}$ & 1 & 1 & 1 & 1 & 1 & 0.4004 \\
$\mathfrak{W} g t$ & 0.1212 & 0.1021 & 0.0213 & 0.0213 & 0.1344 & \\
\hline
\end{tabular}

TABLE IV: Soft set representation of $R S_{2}$

\begin{tabular}{ccccccc}
\hline & $\gamma_{12}$ & $\gamma_{23}$ & $\gamma_{31}$ & $\gamma_{41}$ & $\gamma_{52}$ & $\mathcal{W} C V$ \\
\hline $\mathcal{F} \zeta_{1}$ & 0 & 0 & 0 & 0 & 1 & 0.0213 \\
$\mathcal{F} \zeta_{2}$ & 0 & 1 & 0 & 0 & 1 & 0.1425 \\
$\mathcal{F} \zeta_{3}$ & 1 & 0 & 0 & 0 & 1 & 0.0427 \\
$\mathcal{F} \zeta_{4}$ & 0 & 0 & 0 & 0 & 0 & 0 \\
$\mathcal{F} \zeta_{5}$ & 0 & 0 & 1 & 1 & 0 & 0.0427 \\
$\mathfrak{B} g t$ & 0.0213 & 0.1212 & 0.0213 & 0.0213 & 0.0213 & \\
\hline
\end{tabular}

$\left(\mathcal{S} S_{4}, \mathcal{S} \mathcal{F} \mathcal{A}_{4}\right)=\left\{\mathcal{S} S_{4}\left(\gamma_{31}\right), \mathcal{S} S_{4}\left(\gamma_{42}\right), \mathcal{S} S_{4}\left(\gamma_{43}\right)\right\}$

$\left.\left(\mathcal{S} S_{5}, \mathcal{S} \mathcal{F} \mathcal{A}_{5}\right)=\left\{S S_{5}\left(\gamma_{41}\right), \mathcal{S} S_{5}\left(\gamma_{52}\right)\right)\right\}$

Furthermore, bijective soft sets as per 3 can be given as; $\mathcal{S} S_{1}\left(\gamma_{11}\right)=\left\{\mathcal{F} \zeta_{1}, \mathcal{F} \zeta_{2}\right\} ; \mathcal{S} S_{1}\left(\gamma_{12}\right)=\left\{\mathcal{F} \zeta_{3}\right\}$

$\mathcal{S} S_{1}\left(\gamma_{13}\right)=\left\{\mathcal{F} \zeta_{4}, \mathcal{F} \zeta_{5}\right\} ; \mathcal{S} S_{2}\left(\gamma_{21}\right)=\left\{\mathcal{F} \zeta_{1}, \mathcal{F} \zeta_{4}, \mathcal{F} \zeta_{5}\right\} ;$

$\mathcal{S} S_{2}\left(\gamma_{22}\right)=\left\{\mathcal{F} \zeta_{3}\right\} ; \mathcal{S} S_{2}\left(\gamma_{23}\right)=\left\{\mathcal{F} \zeta_{2}\right\}$

$\mathcal{S} S_{3}\left(\gamma_{31}\right)=\left\{\mathcal{F} \zeta_{5}\right\} ; \mathcal{S} S_{3}\left(\gamma_{32}\right)=\left\{\mathcal{F} \zeta_{3}, \mathcal{F} \zeta_{4}\right\} ;$

$\mathcal{S} S_{3}\left(\gamma_{33}\right)=\left\{\mathcal{F} \zeta_{1}, \mathcal{F} \zeta_{2}\right\} ; \mathcal{S} S_{4}\left(\gamma_{41}\right)=\left\{\mathcal{F} \zeta_{5}\right\}$

$\mathcal{S} S_{4}\left(\gamma_{42}\right)=\left\{\mathcal{F} \zeta_{4}\right\} ; \mathcal{S} S_{4}\left(\gamma_{43}\right)=\left\{\mathcal{F} \zeta_{1}, \mathcal{F} \zeta_{2}, \mathcal{F} \zeta_{3}\right\}$

$\mathcal{S} S_{5}\left(\gamma_{51}\right)=\left\{\mathcal{F} \zeta_{4}, \mathcal{F} \zeta_{5}\right\} ; \mathcal{S} S_{5}\left(\gamma_{52}\right)=\left\{\mathcal{F} \zeta_{1}, \mathcal{F} \zeta_{3}, \mathcal{F} \zeta_{3}\right\} ;$

These relations satisfy the both conditions of bijective soft set. Let us consider $\left(S S_{1}, S F A_{1}\right)$ as an example, union of all the soft sets of $\left(S S, S F A_{2}\right)$ marks concept sources, which is universal set $\mathrm{U}$ or $\bigcup_{\gamma_{i j} \in S F A_{i}} S S\left(\gamma_{1 j}\right)=U$. Furthermore considering any of two SFA values, $\gamma_{11}, \gamma_{12} \in S F A_{1}, \gamma_{11} \neq$ $\gamma_{12}, S S_{1}\left(\gamma_{11}\right) \cap S S_{1}\left(\gamma_{12}\right)=\varnothing$

iv Let us capture the preference values as per 4 to introduce Shannon weights and represent it as $\mathcal{P} D M$. Radio specialists assign preference values as shown in Table I, where; Low $=0.1 ;$ Medium $=0.4 ;$ High $=0.6 ;$ Very high $=0.8$

v Projection value $(p v)$, entropy ( $\leftleftarrows n t)$, divergence (Div), and weight $(\mathfrak{M g} t)$ of each signal attribute value $\gamma_{i j}$ computed as per 5 can be realized in Table II.

vi After computing Shannon weights, we obtain abstract requirements from radio specialists $\mathcal{R} S R$ for Wi-Fi Halow signal coverage in collapsed structures as ;
TABLE V: Soft set representation of $R S_{3}$

\begin{tabular}{ccccccc}
\hline & $\gamma_{11}$ & $\gamma_{21}$ & $\gamma_{32}$ & $\gamma_{41}$ & $\gamma_{52}$ & $\mathcal{W} C V$ \\
\hline $\mathcal{F} \zeta_{1}$ & 1 & 0 & 0 & 0 & 1 & 0.1557 \\
$\mathcal{F} \zeta_{2}$ & 1 & 0 & 0 & 0 & 1 & 0.1557 \\
$\mathcal{F} \zeta_{3}$ & 0 & 1 & 1 & 0 & 1 & 0.05373 \\
$\mathcal{F} \zeta_{4}$ & 0 & 0 & 1 & 0 & 1 & 0.0111 \\
$\mathcal{F} \zeta_{5}$ & 0 & 0 & 0 & 0 & 1 & 0.0213 \\
$\mathfrak{B} g t$ & 0.1344 & 0.0213 & 0.0111 & 0.0213 & 0.0213 & \\
\hline
\end{tabular}

TABLE VI: Ideal and NIS for each $R S$

\begin{tabular}{clcc}
\hline Radio & Specialists & $\mathcal{J} S\left(\gamma_{i}^{*}\right)$ & $\mathcal{N} I S\left(\gamma_{i}^{*}\right)$ \\
\hline & $\Re S_{1}$ & 0.40038 & 0 \\
& $R S_{2}$ & 0.14254 & 0 \\
& $R S_{3}$ & 0.15574 & 0.01105 \\
\hline
\end{tabular}

$\mathcal{R S R}_{1}=\left\{\gamma_{13}, \gamma_{21}, \gamma_{31}, \gamma_{41}, \gamma_{51}\right\}$

$\mathcal{R S R}_{2}=\left\{\gamma_{12}, \gamma_{23}, \gamma_{31}, \gamma_{41}, \gamma_{52}\right\}$

$\mathcal{R S R}_{3}=\left\{\gamma_{11}, \gamma_{22}, \gamma_{32}, \gamma_{41}, \gamma_{52}\right\}$

vii Subsequent tabular soft set representation along-with $\mathcal{W} C V$ of each $\gamma_{i j}$ for respective radio specialists $\mathcal{R} \mathcal{S}$ can be visualized through Table III, IV, and V.

viii After that, we compute IS and NIS for all $\mathcal{R S}$ s using 8 , a key point in TOPSIS and envisage it in Table VI.

ix Corresponding separation measures of each $\mathcal{R S}$ from IS and NIS computed as per 9 can be shown in Table VII, whereas combined separation measure based on signal functional concepts $\mathcal{F} \zeta_{K}$ and afore-computed separation measures is presented in Table VIII.

x Finally, we calculate the relative closeness of each $\mathcal{F} \zeta_{k}$ towards IS as in Table IX and rank the best result. We get $\zeta_{5}$ as our signal functional concept. $₹ \zeta_{5}$ is given as;

$\mathcal{F} \zeta_{5}=\left\{\gamma_{13}, \gamma_{21}, \gamma_{31}, \gamma_{41}, \gamma_{51}\right\}$

Relative values of $\mathcal{S F \mathcal { A }}$ for $\mathcal{F} \zeta_{5}$ are; $\zeta_{5}=\{$ Low, Poor, V.Good,Good,V.Good,Maximum $\}$

Finally, let us analyze the achieved signal functional concept as it may lead to a better solution. We observe from $\zeta_{5}$ that low power, poor MDS threshold, very good coverage, good echo reception probability, and higher debris layer is the best case to study the coverage of Wi-Fi Halow in collapsed structures. But, there can be an argument that radio specialists can directly give their opinions, and there is no need to apply Shannon entropy method. The answer to this argument is that varied opinions of radio specialists cannot 
TABLE VII: Seperation measure of $R S_{1}, R S_{2}, R S_{3}$ from IS and NIS

\begin{tabular}{ccccccc}
\hline $\mathcal{F u n c t i o n a l}$ Concepts & $\Delta_{1 \kappa}^{*}$ & $\Delta_{1 \kappa}^{*}$ & $\Delta_{2 \kappa}^{*}$ & $\Delta_{2 \kappa}$ & $\Delta_{3 \kappa}^{*}$ & $\Delta_{3 \kappa}^{*}$ \\
\hline $\mathcal{F} \zeta_{1}$ & 0.0889709 & 0.01042441 & 0.01468944 & $4.553956 E^{-4}$ & 0 & 0.0209351961 \\
$\mathcal{F} \zeta_{2}$ & 0.1603041 & 0 & 0 & 0.0203176516 & 0 & 0.0209351961 \\
$\mathcal{F} \zeta_{3}$ & 0.1603041 & 0 & $9.97201 E^{-3}$ & $1.82158 E^{-3}$ & 0.014060401 & $1.8215824 E^{-3}$ \\
$\mathcal{F} \zeta_{4}$ & $1.82158 E^{-3}$ & 0.12794929 & 0.0203176516 & 0 & 0.0209351961 & 0 \\
$\mathcal{F} \zeta_{5}$ & 0 & 0.1603041 & $9.97201 E^{-3}$ & $1.82158 E^{-3}$ & 0.01806336 & $1.058841 E^{-4}$ \\
\hline
\end{tabular}

TABLE VIII: Combined seperation measure

\begin{tabular}{ccc}
\hline Functional Concepts & \multicolumn{1}{c}{$\Delta_{\kappa}^{*}$} & $\Delta_{\kappa}$ \\
\hline $\mathcal{F} \zeta_{1}$ & 0.3219632588 & 0.1783676027 \\
$\mathcal{F} \zeta_{2}$ & 0.4003799446 & 0.2031079705 \\
$\mathcal{F} \zeta_{3}$ & 0.4250672301 & 0.06035861496 \\
$\mathcal{F} \zeta_{4}$ & 0.2075437971 & 0.3577 \\
$\mathcal{F} \zeta_{5}$ & 0.1674531606 & 0.402779726
\end{tabular}

TABLE IX: Relative Closeness of $\mathcal{F} \zeta$

\begin{tabular}{cc}
\hline Functional Concepts & $\zeta_{\kappa}^{*}$ \\
\hline $\mathcal{F} \zeta_{1}$ & 0.3564993022 \\
$\mathcal{F} \zeta_{2}$ & 0.3365570929 \\
$\mathcal{F} \zeta_{3}$ & 0.1243415767 \\
$\mathcal{F} \zeta_{4}$ & 0.6328242819 \\
$\mathcal{F} \zeta_{5}$ & 0.7063425058 \\
\hline
\end{tabular}

be trusted directly as it is multicriteria decision-making problem. It also needs cross-verification from wireless communication theory and multiple opinion assessment to reach proper concept. So, Shanon entropy TOPSIS based on bijective soft set method ensures trustworthy conceptual analysis from multicriteria coverage problem.

\section{Coverage Range Methods}

In this section, let us study wireless signal propagation methods that may provide us better coverage. Firstly, we discuss the problem geometry from debris and echo models. After that, we employ TR and Wi-Fi radar techniques that may provide us better echo having proper CSI.

Problem Geometry: Let us revisit the echo model adopted in Fig 2d. We observe that the Wi-Fi transmitter usually radiates signals to possibly every direction. But to map the model for collapsed structure, we only assume the directional pattern of antenna focused towards debris to avoid signals reflected from undesired resources and consider as noises. We also assume that a Wi-Fi device is placed outside of rubble with no other radio device in the vicinity. This placement provides us an understanding that Wi-Fi signal penetration through our debris model will comprise of two parts, as shown in Fig 5a. These are free-space and debris paths. It can be seen that wireless signal will travel about 1 meter in freespace before penetrating debris. After that, it faces many attenuations, fading, and damping from noisy and cluttered environments inside the debris model. A simplified relation from Fig $5 \mathrm{a}$ can be given as follows;

$$
P L=P L_{\text {free-space }}+P L_{\text {debris }}
$$

We can compute free-space path loss as below;

$$
P L_{\text {free-space }}=20 \log _{10}(d)+20 \log _{10}(f)+\log _{10}\left(\frac{4 \pi}{c}\right)-\text { AGains }
$$

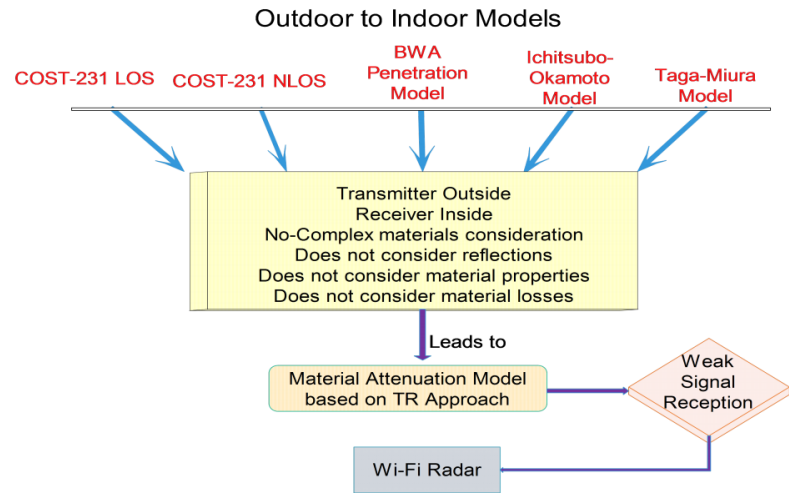

Fig. 4: Path to coverage range methods

where $d$ and $f$ denote free-space distance and frequency respectively. AGains represents the corresponding antenna gains for a Wi-Fi device. Now, the only remaining term is $P L_{\text {debris }}$.

Debris Path Loss: To study debris path loss, we need to consider attenuations; which are caused by reflections, scattering, multipath, diffraction, and fading. Furthermore, fading will be both slow and fast, but to explicitly study attenuation and fading phenomena for collapsed structure, we need another research, and it cannot be covered in this work. So, we only use the existing literature for attenuations and introduce the term, $X_{G}$ that comprises every probable path loss encountered to Wi-Fi signal and is given as below;

$$
\begin{aligned}
P L_{\text {debris }} & =X_{G}=x * A F(B r)+y * A F(C o n) \\
& +z * A F(G l s)+k * A F(\text { Lum })
\end{aligned}
$$

where $x, y, z$, and $k$ stands for the number of layers for our materials. $A F$ is an attenuation factor induced by a single layer of concrete, glass, brick, and lumber, respectively. The thickness of structural elements will also characterize the ramification of layers, and $A F$ values alter correspondingly.

Outdoor to Indoor Models: Wi-Fi Halow signal coverage problem in debris model has resemblance with outdoor to indoor signal penetration scenario. So, we need to look into existing path loss models that only deals with building penetration loss from outdoor to indoor, as shown in Fig 4. These methods are COST-231 LOS, COST-231 NLOS, Broadband wireless access (BWA) model, Ichitsubo-Okamoto, and TagaMiura model using the identification of path passing through wall openings which have been discussed in [29]. We observe that all outdoor to indoor models consider receiver to be inside the building, which is contrary to our approach where the receiver should be outside of debris. Secondly, these methods do not adequately consider attenuations caused by materials 


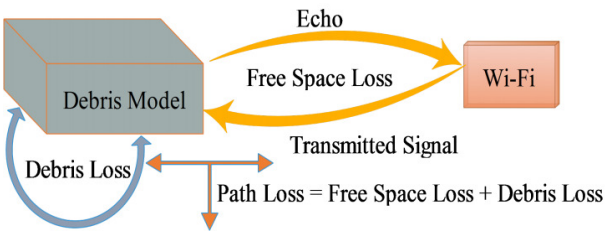

(a) Path Loss in Collapsed structure

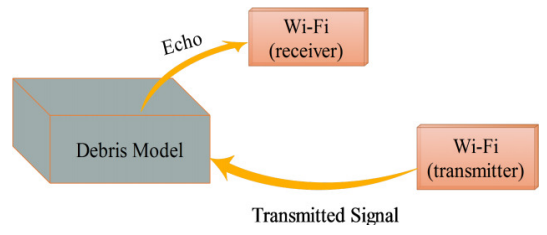

(b) TR-Approach

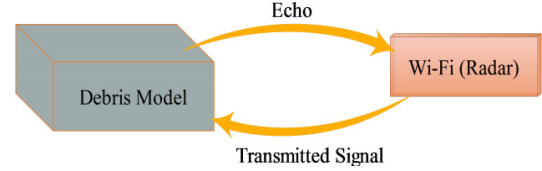

(c) Wi-Fi Radar

Fig. 5: Illustration of coverage range methods

that adversely affect Wi-Fi signal in collapsed structures. So, this triggers us to modify the existing models with materials attenuation approach, which leads to TR and Wi-Fi radar techniques based on the probability of signal strength.

CSI Consideration: This study aims to investigate the coverage of Wi-Fi Halow signal that may be utilized for breathing detection in the future. It is mainly possible due to CSI encompassed in the echo received from debris model. The echo signal will also contain clutter and noises in the CSI that may yield untrustworthy breathing detection. So, there is a need to consider CSI with robust breathing information for both coverage range methods. To achieve this, the echo signal should be above or equal to PDT, as discussed earlier in the echo model.

\section{A. TR-Approach}

In this technique, we make use of a "PL-Collapsed" from our work [30] with extension to more complex structure. We adopt this method to study existing outdoor to indoor path loss methods [29] for coverage in collapsed structures, as mentioned earlier in Fig 4. We observe that even Wi-Fi sensing applications discussed before employ the same propagation models. To the best of our knowledge, all these techniques do not consider receiver to be placed outside although there is one commonality that both transmitter and receiver at two different locations. Although, this mode also does not work well in collapsed structures, yet we still need to study the modified TR-approach for possible coverage as it can pave the way for new techniques. So, let us revisit the echo signal for the TR approach as shown in Fig 5b, where transmitter and receiver are placed at two distinct positions. To realize the Wi-Fi signal penetration in debris model, we consider the link budget equation as;

$$
P_{r}=G_{t}+G_{r}+P_{t}+-P L
$$

where $G$ and $P$ represent antenna gains and power for transceivers. $P L$ defines the path losses incurred to $\mathrm{Wi}-\mathrm{Fi}$ signal in both free-space and debris. Let us compute path loss which is mainly dependent on the distance covered in debris and can be represented in the log as below;

$$
P L=P L_{\text {free-space }}+10 * \alpha * \log _{10}\left(\frac{d}{d_{0}}\right)+X_{g}
$$

where $P L\left(d_{0}\right)$ is path loss for first 1 meter $d_{0}$. But this is only calculated for outside of debris as after penetrating the collapsed structure, there is no free space. $\alpha$ is path loss exponent having values ranging from 2 to 6 depending to the nature of the environment. We consider it 5 so deal with complex structures while excluding metallic materials. Finally, we can incorporate attenuations of multiple layers of our debris through $X_{G}$.

CSI Consideration for TR Approach: To achieve an echo having substantial CSI, we consider a minimum detectable signal (MDS). We envisage that echo should be equal or above than $M D S$ with the probability of acceptable error rate $\operatorname{MDS}\left(P_{e}\right)$ and can be given as below;

$$
P_{t x}+G_{t x}+G_{r x}-P L \geq \operatorname{MDS}\left(P_{e}\right)
$$

\section{B. Wi-Fi Radar}

In this method, we employ $\mathrm{Wi}-\mathrm{Fi}$ radar technique to complex structures, as shown in Fig 5c. In this technique, transmitter and receiver are considered to be at the same location quite similar to radar. We are confident that it will yield better results than traditional TR-approach by reducing clutter and noise. We envision that all the existing studies do not study the Wi-Fi radar path loss model for debris scenario except [33], which is work of authors, where they discussed it for simple structures without comparison with TR-approach. Let us revisit $\mathrm{Wi}-\mathrm{Fi}$ radar for our debris model as discussed in SectionII-B. Under normal circumstances, it depends on crosssectional area, signal intensities, antenna gains and distance between target and transmitter. It can be given as follows;

$$
P_{r}=P_{t} G_{t} G_{r}\left(\frac{c^{2} \sigma}{(4 \pi)^{3} f^{2} R^{4}}\right)
$$

where $P_{t}, P_{r}, G_{t}$, and $G_{r}$ represents power and gains of Wi-Fi radar transceiver. $\sigma$ is cross-sectional area, which we consider as debris area while $f$ and $R$ symbolizes the frequency and range correspondingly. After this, the received signal at the input of Wi-Fi radar will be;

$$
\left\{\begin{array}{c}
\text { WiFi Signal received } \\
\text { at WiFi Radar }
\end{array}\right\}=\frac{P_{t} G_{t} \lambda^{2} 4 \pi \sigma}{(4 \pi R)^{2} \lambda^{2}} \times \frac{G_{r} \lambda^{2}}{(4 \pi R)^{2}}
$$

Although, a detailed derivation of $\mathrm{Wi}-\mathrm{Fi}$ radar range is provided in annexure A, yet the reduction of (8) to log form after doing some mathematics can be as follows;

$$
P_{R}=G_{T}+G_{R}+G_{\sigma}+P_{T}-2 \beta_{1}
$$

where $\beta_{1}$ and $G_{\sigma}$ represents free-space path losses and target gain respectively. Now, we need to incorporate the debris losses and clutter faced by Wi-Fi Halow because of multilayered scenario from (3) in (9), and then we reconvert log form to normal scale given as below;

$$
\text { WiFiRadar Range }=R=\sqrt[4]{\frac{P_{t} G_{t} G_{r}}{P_{r}}\left(\frac{\lambda^{2} \sigma}{(4 \pi)^{3}}\right) \frac{1}{X_{g}}}
$$


TABLE X: Simulation Parameters

\begin{tabular}{|l|c|}
\hline & Values \\
Parameter & $(902,779) \mathrm{MHz}$ \\
Operational Frequencies & $(30,24,23,13,10) \mathrm{dBm}$ \\
Transmission Power & $(6,12,13,23,26) \mathrm{dBi}$ \\
Antenna Gains & $4 m^{2}$ \\
Radar Cross Section & Concrete, Brick, Glass, Lumber \\
Debris Type & $23 \mathrm{~dB}$ at $902 \mathrm{MHz}$ \\
Attenuation of Concrete 8" & $7 \mathrm{~dB}$ at $902 \mathrm{MHz}$ \\
Attenuation of Brick 10.5" & $2 \mathrm{~dB}$ at $902 \mathrm{MHz}$ \\
Attenuation of Glass (13mm) & $2.8 \mathrm{~dB}$ at $902 \mathrm{MHz}$ \\
Attenuation of Lumber (76mm) & $10(4 \mathrm{~b}+2 \mathrm{c}+2 \mathrm{~g}+21)$ \\
Minimum Debris Layers & $13(5 \mathrm{~b}+3 \mathrm{c}+2 \mathrm{~g}+31)$ \\
& $18(8 \mathrm{~b}+3 \mathrm{c}+3 \mathrm{~g}+41)$ \\
Maximum Debris Layers & $22(10 \mathrm{~b}+4 \mathrm{c}+4 \mathrm{~g}+41)$ \\
& $1 \mathrm{MHz}, 16 \mathrm{QAM}$ \\
Modulation Type & $1 \mathrm{MHz}$ \\
Channel BW at 256QAM & $16 \mathrm{MHz}, 1 \mathrm{MHz}$ \\
Channel BW at 64QAM & $-70 \mathrm{dBm}$ \\
Channel BW at 16QAM & $-77 \mathrm{dBm},-78 \mathrm{dBm}$ \\
MDS Threshold at 256QAM & $-71 \mathrm{dBm},-75 \mathrm{dBm}$ \\
MDS Threshold at 64QAM & 5 \\
MDS Threshold at 16QAM & \\
Path Loss Exponent &
\end{tabular}

After getting a complete relation of coverage from (10), we can take (10) to log form again for final range calculation as provided in A. We consider $\sigma$ in (10) as debris area under observation whereas $P_{r}$ is the minimum detectable signal which can provide us desired echo.

CSI Consideration for Wi-Fi Radar: To achieve an echo having desired CSI for Wi-Fi radar, we consider signal to noise ratio (SNR). We envision that received echo should have SNR above or equal to a pre-defined threshold as $S N R \geq S N R_{T}$.

\section{NUMERICAL RESULTS}

In this section, we present our simulation method and numerical results for evaluation. We simulated Wi-Fi Halow for vital signal attributes. Finally, we compare these results to select the best radio propagation method for better coverage in a collapsed structure.

\section{A. Method}

To achieve substantial results, there is an immense need to select the simulation parameters shown in Table X accurately. We have performed simulations in MATLAB $18 \mathrm{a}$.

Let us discuss the reasons for the selection of these simulation parameters. We considered two operating frequencies for Wi-Fi Halow i-e., China(779MHz) and the US(902MHz), as given in [34] [45], which are regulated by IEEE Task Group [45]. We selected China and US standards due to our field survey and for its worldwide acceptance. Now, both countries have their own power consideration for Wi-Fi Halow spectrum limits as in [45]. Similarly, variance in other parameters such signal intensities and antenna gains are regulated with Federal Communications Commission(FCC $)^{3}$ para 17.245 rules for PTMP (Point to multi-point) ${ }^{4}$.

Next, we discuss the selection of debris type. We considered concrete, brick, glass, and lumber for our simulations because

\footnotetext{
${ }^{3} \mathrm{https}: / /$ www.fcc.gov/tags/radio-rules

${ }^{4} \mathrm{https}: / /$ www.air802.com/fcc-rules-and-regulations.html
}

these were the primary structural materials found in the field survey. These materials have different thickness level, but to simplify the results, we only considered brick $10.5^{\prime \prime}$, concrete 8 ", lumber $(76 \mathrm{~mm})$, and glass $(13 \mathrm{~mm})$. The depth of these materials defines the complexity of collapsed structures. We employ the attenuations caused by single layers of under considered materials from Digi ${ }^{5}$ and U.S. National Institute of Standards and Technology(NIST) ${ }^{6}$. We also selected the debris layers with minimum as $(10,13)$ and maximum as $(18$, 22) to properly compute the Wi-Fi Halow signal penetration through debris model.

Finally, we discuss the need for an MDS threshold, which is very important for better echo reception. We have taken it from $[34,45]$ ranging $-70 \mathrm{dBm}$ to $-78 \mathrm{dBm}$ depending on three QAM types $(256,64,16)$ with $1 \mathrm{MHz}$ channel bandwidth. It is evident that higher QAM ensures better data rate, but also cause noise and interference. That is why we have selected both high and low QAM scenario to have a better knowledge of Wi-Fi Halow signals. Path loss exponent is taken as 5 to consider the complex debris layout.

\section{B. Results}

We conducted simulations on the criteria as mentioned earlier by mapping field survey to theory. We present simulations results in Table XI and XII. These results show coverage level at varied debris type across antenna and intensity for $\mathrm{Wi}-\mathrm{Fi}$ Halow frequencies. Both frequency cases have been tested for overall 240 cases to have a better inference. Let us compare the coverage results in subsequent sections. For convenience, we have presented TR and Wi-Fi radar methods as 1 and 2 in the graphical results.

1) Range w.r.t Debris Type: We classify debris into two types for the layered model. We consider type 1 as (MinLayer=10, MaxLayer=18) followed by type 2 as (MinLayer=10, MaxLayer=18) and perform simulations for $902 \mathrm{MHz}$ and $779 \mathrm{MHz}$. A comparison is as follows;

a) $902 \mathrm{MHz}$ : We compare the results for type 1 and 2 debris from Table XI and plot them as individual value plot in Fig 6 and 7. It can be seen from results that type 1 debris can provide more coverage than the other one. Let us revisit Table XI for $10 \mathrm{dBm}$ power case, we can observe that range for type $1 \mathrm{~min}$ layer is $11.916 \mathrm{~m}$, whereas, for type $2 \mathrm{~min}$ layer, the range is $2.63 \mathrm{~m}$. This behavior is the same across all other power values. We observe that increasing debris layers reduces coverage.

b) $779 \mathrm{MHz}$ : Similarly, we compare the results for type 1 and 2 debris from Table XII and plot them as individual value plot in Fig 8 and 9 . We get the similar trend to that of $902 \mathrm{MHz}$. Now, again looking into Table XII for $10 \mathrm{dBm}$ power case, we can observe that range for type 1 min layer is $12.63 \mathrm{~m}$, whereas, for type $2 \mathrm{~min}$ layer, the range is $2.79 \mathrm{~m}$. This behavior is the same across all other power values. Quite similar to $902 \mathrm{MHz}$, we observe that increasing debris layers reduces coverage. But we observe that $779 \mathrm{MHz}$ has slightly better coverage than $902 \mathrm{MHz}$.

\footnotetext{
${ }^{5} \mathrm{http}: / / \mathrm{ftp} 1$.digi.com/support/images/XST-AN005a-IndoorPathLoss.pdf

${ }^{6} \mathrm{http}: / /$ www.eiwellspring.org/shielding.html
} 
TABLE XI: Wi-Fi Halow signal coverage at $902 \mathrm{MHz}$

\begin{tabular}{|c|c|c|c|c|c|c|c|c|c|c|c|c|c|}
\hline \multirow{4}{*}{$\begin{array}{c}\text { Transmit } \\
\text { Power } \\
\text { dBm }\end{array}$} & \multirow{4}{*}{$\begin{array}{c}\text { Max } \\
\text { Antenna } \\
\text { dBi }\end{array}$} & \multicolumn{12}{|c|}{ Range in Collapsed Structure (m) } \\
\hline & & \multicolumn{4}{|c|}{ QAM 256(R $=5 / 6)(\mathrm{MS}=-70 \mathrm{dBm})$} & \multicolumn{4}{|c|}{ QAM $16(\mathrm{R}=3 / 4)(\mathrm{MS}=-71 \mathrm{dBm})$} & \multicolumn{4}{|c|}{ QAM $16(\mathrm{R}=3 / 4)(\mathrm{MS}=-75 \mathrm{dBm})$} \\
\hline & & \multicolumn{2}{|c|}{$\mathrm{T}-\mathrm{R}$} & \multicolumn{2}{|c|}{ Wi-Fi Radar } & \multicolumn{2}{|c|}{$\mathrm{T}-\mathrm{R}$} & \multicolumn{2}{|c|}{ Wi-Fi Radar } & \multicolumn{2}{|c|}{$\mathrm{T}-\mathrm{R}$} & \multicolumn{2}{|c|}{ Wi-Fi Radar } \\
\hline & & Mn 10 & Mx 18 & Mn 10 & Max 18 & Mn 10 & Mx 18 & Mn 10 & Mx 18 & Mn 10 & Mx 18 & Mn 10 & Mx 18 \\
\hline 30 & 6 & 0.7519 & 0.0506 & 4.4289 & 0.1518 & 0.7873 & 0.0530 & 4.6913 & 0.1518 & 0.9465 & 0.0637 & 5.9060 & 0.1518 \\
\hline 24 & 12 & 1.7224 & 0.1159 & 6.2560 & 0.2144 & 1.8036 & 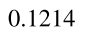 & 6.6 & 0.2144 & 2.1684 & 0.1 & 8.3425 & 0.2144 \\
\hline \multirow[t]{2}{*}{23} & 13 & 1.9776 & 0.1331 & 6.6267 & 0.2271 & 2.0708 & 0.1394 & 7.0193 & 0.2271 & 2.4897 & 0.1675 & 8.8368 & 0.2271 \\
\hline & & & & & & \multicolumn{4}{|c|}{ QAM64(R =3/4)(MS=-78dBm) } & \multicolumn{4}{|c|}{ QAM64(R =5/6)(MS=-77dBm) } \\
\hline \multirow{4}{*}{$\begin{array}{l}13 \\
10 \\
\end{array}$} & 23 & 7.8730 & 0.5298 & 11.7840 & 0.4039 & 11.3799 & 0.7658 & 18.6764 & 0.4039 & 10.8678 & 0.7314 & 17.6317 & 0.4039 \\
\hline & 26 & 11.9163 & 0.8019 & 14.0054 & 0.4801 & 17.2242 & 1.1592 & 22.1970 & 0.4801 & 16.4490 & 1.1070 & 20.9553 & 0.4801 \\
\hline & & & & & & \multicolumn{4}{|c|}{ QAM $16(\mathrm{R}=3 / 4)(\mathrm{MS}=-71 \mathrm{dBm})$} & \multicolumn{4}{|c|}{ QAM 16(R =3/4)(MS=-75dBm) } \\
\hline & & Mn 13 & Mx 22 & Mn 13 & $\operatorname{Max} 22$ & Mn 13 & Mx 22 & Mn 13 & Mx 22 & Mn 13 & Mx 22 & Mn 13 & Mx 22 \\
\hline 30 & 6 & 0.1660 & 0.00 & 0 & 0.0 & 8 & 0.0 & 0.7 & 0.0 & 0.2 & 0.0 & & 0.0161 \\
\hline 24 & 12 & 0.3803 & 0.0192 & 0.9469 & 0.0227 & 0.3982 & 0.02 & 1.00 & 0.02 & 0.4788 & 0.0242 & 1.2 & 0.0227 \\
\hline \multirow[t]{2}{*}{23} & 13 & 0.4367 & 0.0221 & 1.0030 & 0.0241 & 0.4572 & 0.0231 & 1.0624 & 0.0241 & 0.5497 & 0.0278 & 1.3357 & 0.0241 \\
\hline & & & & & & \multicolumn{4}{|c|}{ QAM64 $(\mathrm{R}=3 / 4)(\mathrm{MS}=-78 \mathrm{dBm})$} & \multicolumn{4}{|c|}{ QAM64 $(\mathrm{R}=5 / 6)(\mathrm{MS}=-77 \mathrm{dBm})$} \\
\hline 13 & 23 & 1.7384 & 0.0879 & 1.7836 & 0.0428 & 2.5 & 0.12 & 2.8 & 0.0428 & & 0.1 & 2.6 & 0.0428 \\
\hline 10 & 26 & 2.6311 & 0.1331 & 2.1198 & 0.0509 & 3.8031 & 0.1924 & 3.3597 & 0.0509 & 3.6320 & 0.1837 & 3.1717 & 0.0509 \\
\hline
\end{tabular}

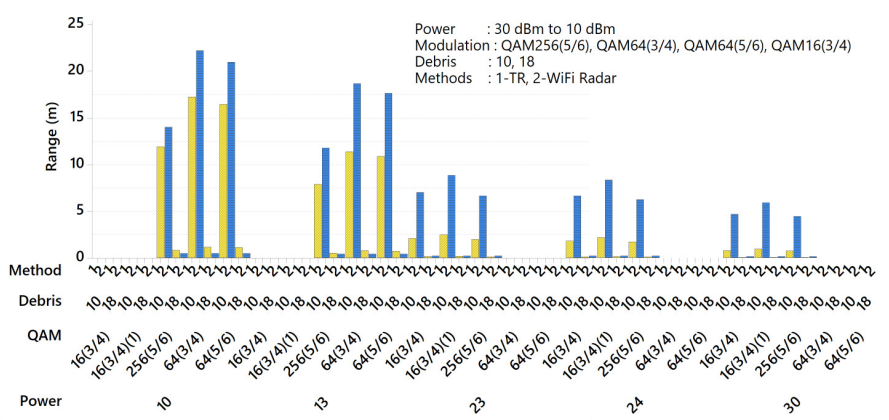

Fig. 6: Range in type 1 debris $(902 \mathrm{MHz})$

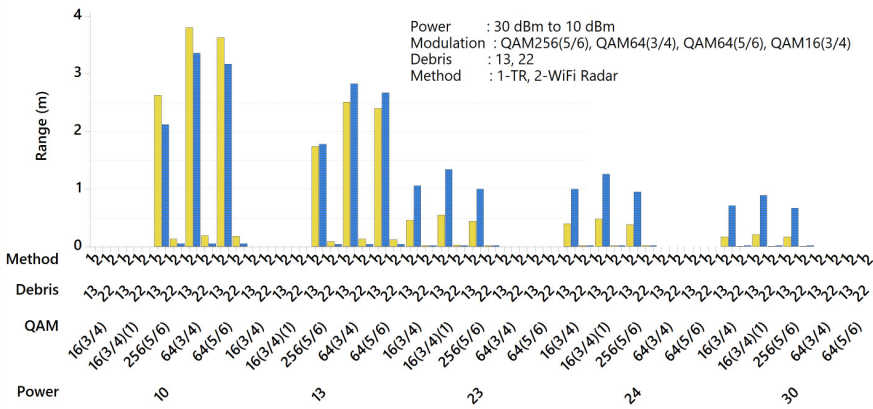

Fig. 7: Range in type 2 debris (902 MHz)

2) Range w.r.t Low Power: We observe from section VI-B1 that low power Wi-Fi Halow yields better results. So, let us focus on $13 \mathrm{dBm}$ and $10 \mathrm{dBm}$ power values for both $902 \mathrm{MHz}$ and $779 \mathrm{MHz}$ as plotted in Fig 10 and 11. It can be observed that $10 \mathrm{dBm}$ with Wi-Fi radar method performs better than $13 \mathrm{dBm}$, e.g., at the $902 \mathrm{MHz}$ range at $13 \mathrm{dBm}$ with QAM 256 and min layer 10 is $11.78 \mathrm{~m}$ using Wi-Fi radar whereas, it is

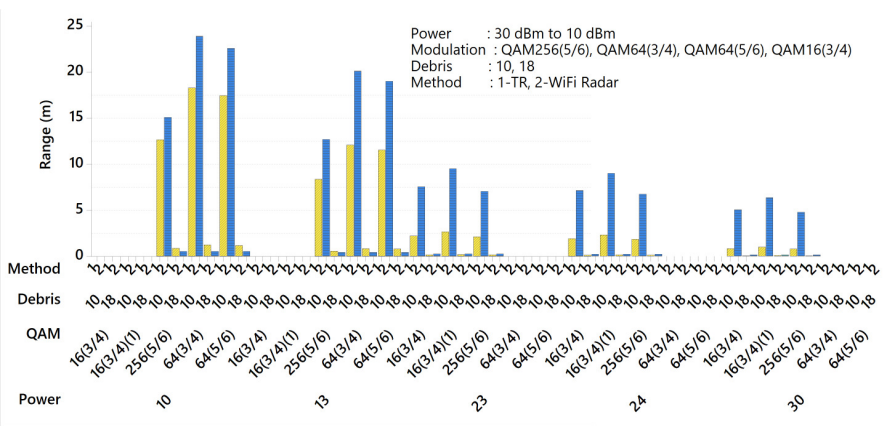

Fig. 8: Range in type 1 debris $(779 \mathrm{MHz})$

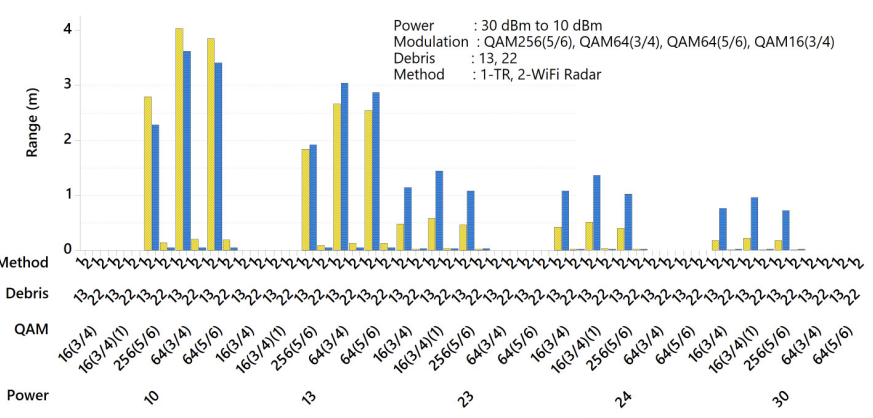

Fig. 9: Range in type 2 debris $(779 \mathrm{MHz})$

$14.0054 \mathrm{~m}$ at $10 \mathrm{dBm}$. There is the same trend as $779 \mathrm{MHz}$. We also observe a decreasing trend of coverage with the increase in debris layers. Let us consider coverage at $779 \mathrm{MHz}$ with 13 min layer, Wi-Fi radar, and $10 \mathrm{dBm}$ power; it is $2.28 \mathrm{~m}$. On the contrary, it is $0.0547 \mathrm{~m}$ with same parameters except for change of debris layers to 22 . 
TABLE XII: Wi-Fi Halow signal coverage at $779 \mathrm{MHz}$

\begin{tabular}{|c|c|c|c|c|c|c|c|c|c|c|c|c|c|}
\hline \multirow{4}{*}{$\begin{array}{c}\text { Transmit } \\
\text { Power } \\
\mathrm{dBm}\end{array}$} & \multirow{4}{*}{$\begin{array}{c}\text { Max } \\
\text { Antenna } \\
\text { dBi }\end{array}$} & \multicolumn{12}{|c|}{ Range in Collapsed Structure (m) } \\
\hline & & \multicolumn{4}{|c|}{ QAM $256(\mathrm{R}=5 / 6)(\mathrm{MS}=-70 \mathrm{dBm})$} & \multicolumn{4}{|c|}{ QAM $16(\mathrm{R}=3 / 4)(\mathrm{MS}=-71 \mathrm{dBm})$} & \multicolumn{4}{|c|}{ QAM $16(\mathrm{R}=3 / 4)(\mathrm{MS}=-75 \mathrm{dBm})$} \\
\hline & & \multicolumn{2}{|c|}{$\mathrm{T}-\mathrm{R}$} & \multicolumn{2}{|c|}{ Wi-Fi Radar } & \multicolumn{2}{|c|}{$\mathrm{T}-\mathrm{R}$} & \multicolumn{2}{|c|}{ Wi-Fi Radar } & \multicolumn{2}{|c|}{$\mathrm{T}-\mathrm{R}$} & \multicolumn{2}{|c|}{ Wi-Fi Radar } \\
\hline & & Mn 10 & Mx 18 & Mn 10 & $\operatorname{Max} 18$ & Mn 10 & Mx 18 & $\mathrm{Mn} 10$ & Mx 18 & Mn 10 & Mx 18 & Mn 10 & Mx 18 \\
\hline 30 & 6 & 0.7973 & 0.0537 & 4.7657 & 0.1634 & 0.8348 & 0.0562 & 5.0481 & 0.1634 & 1.0037 & 0.0675 & 6.3552 & 0.1634 \\
\hline 24 & 12 & 1.8264 & 0.1229 & 6.7318 & 0.2307 & 1.9125 & 0.1 & 7.1306 & 0.2307 & 2.2994 & 0.1547 & 8.9769 & 0.2307 \\
\hline \multirow[t]{2}{*}{23} & 13 & 2.0970 & 0.1411 & 7.0306 & 0.2444 & 2.1959 & 0.1478 & 7.5532 & 0.2444 & 2.6400 & 0.1777 & 9.5089 & 0.2444 \\
\hline & & & & & & \multicolumn{4}{|c|}{ QAM64 $(\mathrm{R}=3 / 4)(\mathrm{MS}=-78 \mathrm{dBm})$} & \multicolumn{4}{|c|}{$\mathrm{QAM} 64(\mathrm{R}=5 / 6)(\mathrm{MS}=-77 \mathrm{dBm})$} \\
\hline \multirow{4}{*}{$\begin{array}{l}13 \\
10\end{array}$} & 23 & 8.3485 & 0.5618 & 12.6803 & 0.4346 & 12.0672 & 0.8121 & 20.0969 & 0.4346 & 11.5241 & 0.7755 & 18.9727 & 0.4346 \\
\hline & 26 & 12.6359 & 0.8504 & 15.0705 & 0.5166 & 18.2645 & 1.2292 & 23.8852 & 0.5166 & 17.4425 & 1.1738 & 22.5491 & 0.5166 \\
\hline & & & & & & \multicolumn{4}{|c|}{ QAM $16(\mathrm{R}=3 / 4)(\mathrm{MS}=-71 \mathrm{dBm})$} & \multicolumn{4}{|c|}{ QAM $16(\mathrm{R}=3 / 4)(\mathrm{MS}=-75 \mathrm{dBm})$} \\
\hline & & Mn 13 & Mx 22 & Mn 13 & Max 22 & Mn 13 & Mx 22 & Mn 13 & Mx 22 & Mn 13 & Mx 22 & Mn 13 & Mx 22 \\
\hline 30 & 6 & 0.1760 & 0.0089 & 0.72 & 0.01 & 0.1843 & 0.0093 & 0.7641 & 0.0173 & 0.2216 & 0.0112 & 0.9619 & 0.0173 \\
\hline 24 & 12 & 0.4033 & 0.0204 & 1.0189 & 0.0244 & 0.4223 & 0.0 & 1.0 & 0.0 & 0.5077 & 0.0257 & 1.3587 & 0.0244 \\
\hline \multirow[t]{2}{*}{23} & 13 & 0.4630 & 0.0234 & 1.0793 & 0.0259 & 0.4849 & 0.0245 & 1.1432 & 0.0259 & 0.5829 & 0.0295 & 1.4392 & 0.0259 \\
\hline & & & & & & \multicolumn{4}{|c|}{ QAM64 $(\mathrm{R}=3 / 4)(\mathrm{MS}=-78 \mathrm{dBm})$} & \multicolumn{4}{|c|}{ QAM64 $(\mathrm{R}=5 / 6)(\mathrm{MS}=-77 \mathrm{dBm})$} \\
\hline 13 & 23 & 1. & 0.0932 & 1.9192 & 0.0460 & 2 & & & & & & & 0.0460 \\
\hline 10 & 26 & 2.7900 & 0.1411 & 2.2810 & 0.0547 & 4.0328 & 0.2040 & 3.6152 & 0.0547 & 3.8513 & 0.1948 & 3.4129 & 0.0547 \\
\hline
\end{tabular}

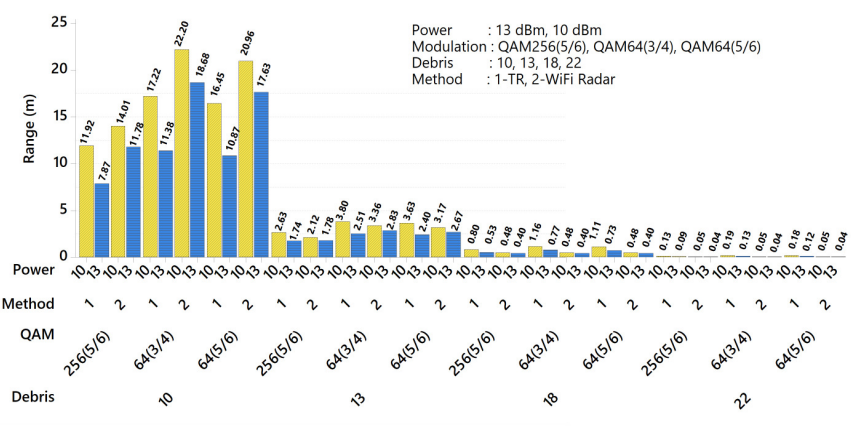

Fig. 10: Range at low power with all debris cases $(902 \mathrm{MHz})$

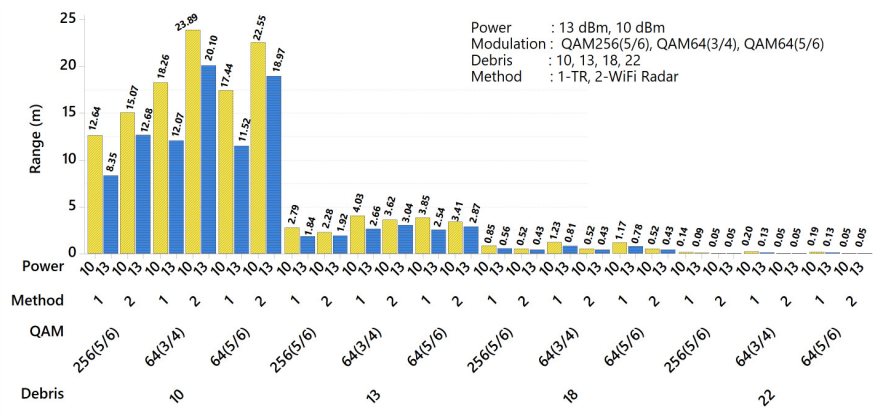

Fig. 11: Range at low power with all debris cases $(779 \mathrm{MHz})$

3) Range w.r.t Frequency: Let us further investigate the low power penetration into debris with a comparison of both frequencies at lower debris layers. We observed from section VI-B2 that lower debris layers can yield better results; so, we envisaged that higher debris layers could not provide better coverage. We plot a frequency-wise comparison for $13 \mathrm{dBm}$ and $10 \mathrm{dBm}$ in Fig 12 and 13 respectively. Let us

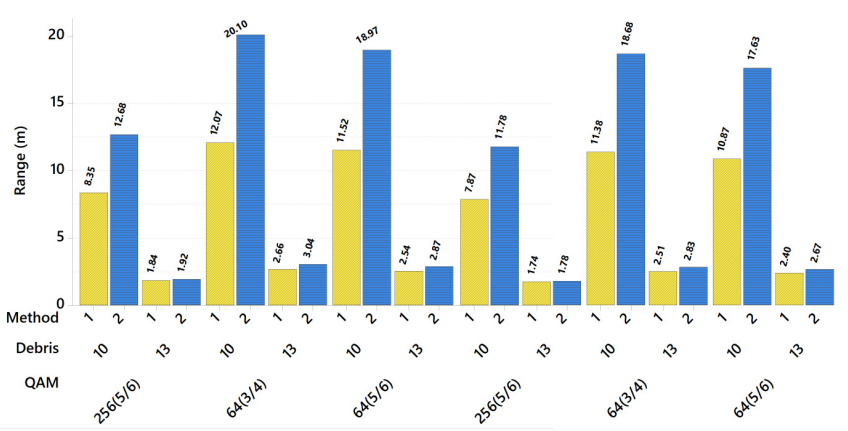

Fig. 12: Frequency wise range for lower debris at $13 \mathrm{dBm}$

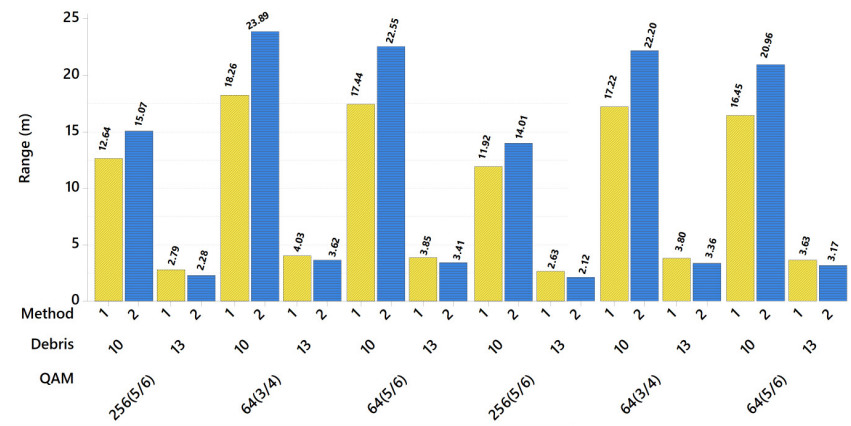

Fig. 13: Frequency wise range for lower debris at $10 \mathrm{dBm}$

consider $10 \mathrm{dBm}$ plot where maximum coverage with 10layered debris employing Wi-Fi radar method at $779 \mathrm{MHz}$ has coverage of $23.89 \mathrm{~m}$ whereas its $22.20 \mathrm{~m}$ for $902 \mathrm{MHz}$. By making a comparison of all results, we figure out that Chinese (779MHz) provides better results than the US $(902 \mathrm{MHz})$.

4) Range w.r.t Method: Finally, we compare the Wi-Fi signal propagation methods. We employed two techniques i- 
e,. TR approach and Wi-Fi radar in this study. To have a comparison, let us consider Fig 13. The maximum coverage range at 10 -layered debris for $779 \mathrm{MHz}$ is $23.89 \mathrm{~m}$ through WiFi radar approach; however, it is 18.26 with a TR approach. From this, we figure out Wi-Fi radar which is a modified form of conventional Doppler radar provides us better coverage. If we compare these methods across both Table XI and XII, we get the same trend providing us immense insight into the application of Wi-Fi radar.

\section{PERFORMANCE ANALYSIS}

In this section, we briefly analyze our study. Firstly, we deliberate the complexity of the problem in the context of existing literature. Then, we present signal propagation methods from both conceptual and signal level. Finally, we discuss the importance, applications, implications, and limitations of this study.

Let us analyze the complexity of the problem. We observe that collapsed structures turn into complex indoor layout having multi-layered obstacles where signal penetration becomes very poor. That is why there are no successful post-disaster rescue solutions available, in spite of the IoT era. Although, there are some rescue techniques, out of which radar sensing [8] is the most promising one, yet it is not well-suited for rescue, except providing a strong motivation for the wireless signal application. Another reason for the need of this study is that most of the existing studies, as discussed in section I-A are expensive and cannot be deployed in developing countries. So, this motivated us to investigate this subject using Wi-Fi radios as sensors in [30]-[33] to initiate a new dimension of research which is quite ubiquitous and cost-effective, and availability is also ensured with the growing internet market.

Let us discuss the methods to study the coverage of Wi-Fi Halow in collapsed structures. We presented signal propagation models both at the conceptual and communication level by employing Shannon entropy TOPSIS and coverage range methods respectively. TOPSIS using Shannon entropy based on bijective soft set was introduced by [46] for multi-criteria decision-making problem. We applied this method to select the best coverage concept from available intensities, gains, thresholds, debris nature, and CSI consideration. Similarly, coverage range methods are further classified into the TR approach and Wi-Fi radar which we earlier introduced separately in [30, 33] for regular debris.

Now, let us discuss the best coverage concept for WiFi Halow signal. Shannon entropy method in section IV provides us immense insight about conceptual coverage where functional concept $\mathcal{F} \zeta_{5}$ was considered as the most optimal solution. $\mathcal{F} \zeta_{5}$ represents low-power, weak MDS threshold, higher echo reception probability, and better debris types. Now, we need to compare this functional concept with simulation results of coverage range methods. After an in-depth analysis of the Section VI, we observe that $\mathrm{Wi}-\mathrm{Fi}$ radar with low power can yield almost the same results to the conceptual level model with a minor difference of debris complexity. Although, there can be an argument on the coverage of both TR and Wi-Fi radar, yet echo reception probability of later is better because in TR approach, received signal only need to be reflected but it does not ensure the same Wi-Fi device. So, there is a probability of more noises for the TR approach.

Finally, we discuss the possible applications of this study. We observe that Wi-Fi sensing application for breathing detection in [18] along-with [14] can provide us better results for rescue if signal strength can be increased using our WiFi radar technique that may give us better CSI. To the best our understandings, all the Wi-Fi sensing applications does not employ Wi-Fi radar method which lacks their practical consideration for collapsed structures. So, we envision that our study can provide a breakthrough to the scientific community to investigate more about Wi-Fi Halow signals in a collapsed structure. It is because of low-frequency, low-power, ubiquitous and cost-effectiveness of Wi-Fi Halow, which is primarily designed for IoTs. And this study of ours justifies that wireless signal can have better coverage using low-power and low-frequency. So, we can rightly say that further research in this domain will lead us to the ubiquitous post-disaster rescue, which will be available in developing countries as well hence truly serving humanity.

Limitations: Like every other work, this study has some limitations. The first and the most important one is a lack of practical implementation of Wi-Fi Halow signals to collapsed structures. It is due to the primitive nature of this study, as we cannot deploy the solution without having proper simulations and conceptual models based on strong theoretical background. So, our work emphasizes the theoretical aspect of the problem. Howsoever, we conducted a field survey to have practical consideration of theory. Another limitation of this study is that we did not discuss fading models and just used attenuation factors for materials from NIST. The reason for this limitation is quite similar that we cannot deal with these issues in simulations directly. So, the best way is to use the practical results of standard bodies. Based on these observations, we are hopeful that this study will trigger further research in IoTs based rescue solutions.

\section{CONCLUSION}

In this work, we studied the feasibility of Wi-Fi Halow for adequate coverage in collapsed structures. To achieve this, we conducted a field survey of an earthquake site to realize debris as multi-obstacle layered indoor layout that needs better signal penetration. After that, we presented signal propagation methods both at a conceptual level and signal communication as well. The first method is based on bijective soft set with an integrated approach of Shannon entropy TOPSIS, which provides results with strong mathematical foundations. Furthermore, the TR approach and Wi-Fi radar techniques present the communication of signals in the debris model to realize the collapsed structure and give us probable coverage. Finally, we conclude that Wi-Fi radar method is the most suited technique as the TR approach can face more attenuations and also it does not seem practical for the collapsed environment. We envision that Wi-Fi Halow with Wi-Fi radar method can trigger more research in this post-disaster rescue. 


\section{APPENDIX A}

PROOF OF THE WI-FI RADAR RANGE EQUATION

$$
P_{r}=\frac{P_{t} G_{t} G_{r} \lambda^{2} \sigma}{(4 \pi)^{3} R^{4}}
$$

Since $\lambda=\frac{c}{f}$; so, (11) can be modified as;

$$
\begin{aligned}
& P_{r}=P_{t} G_{t} G_{r}\left(\frac{c^{2} \sigma}{(4 \pi)^{3} f^{2} R^{4}}\right) \\
& \quad\left\{\begin{array}{c}
\text { Signal Received } \\
\text { at Debris }
\end{array}\right\}=\frac{P_{t} G_{t} G_{r} \lambda^{2}}{(4 \pi R)^{2}}
\end{aligned}
$$

Since $G_{r}=\frac{4 \pi \sigma}{\lambda^{2}}$, so signal reflected from debris will be;

$$
\begin{aligned}
\left\{\begin{array}{c}
\text { Signal Reflected } \\
\text { from Debris }
\end{array}\right\} & =\frac{P_{t} G_{t} \lambda^{2} 4 \pi \sigma}{(4 \pi R)^{2} \lambda^{2}} \text { then } \\
& \left\{\begin{array}{c}
\text { Received Signal } \\
\text { at WiFi Radar }
\end{array}\right\}=\frac{P_{t} G_{t} \lambda^{2} 4 \pi \sigma}{(4 \pi R)^{2} \lambda^{2}} \times \frac{G_{r} \lambda^{2}}{(4 \pi R)^{2}}
\end{aligned}
$$

Let us reduce above equation in to $\log$ form as;

$$
\begin{aligned}
& P_{R}=P_{T}+G_{T}+G_{R}+20 \log \left(\frac{\lambda}{4 \pi R}\right)+10 \log \left(\frac{4 \pi \sigma}{\lambda^{2}}\right)+20 \log \left(\frac{\lambda}{4 \pi R}\right) \\
& \text { where } \beta_{1}=20 \log \left(\frac{4 \pi f R}{c}\right) \text { and } G_{\sigma}=10 \log \left(\frac{4 \pi \sigma}{\lambda^{2}}\right) \\
& P L=P L_{\text {free-space }}+P L_{\text {debris }}
\end{aligned}
$$

To compute coverage, we need to reconvert log form and employ( 12) which provides us range in power form.

$$
R^{4}=\frac{P_{t} G_{t} G_{r}}{P_{r}}\left(\frac{\lambda^{2}}{4 \pi}\right)\left(\frac{4 \pi \sigma}{\lambda^{2}}\right)\left(\frac{\lambda^{2}}{4 \pi}\right) \frac{1}{X_{g}}
$$

Finally, by re-arranging the above equation for range, we get;

$$
\text { WiFiRadar Range }=R=\sqrt[4]{\frac{P_{t} G_{t} G_{r}}{P_{r}}\left(\frac{\lambda^{2} \sigma}{(4 \pi)^{3}}\right) \frac{1}{X_{g}}}
$$

(18) in log form can be given as follows;

$$
\begin{aligned}
10 \log R & \cong \frac{1}{4}\left[P_{T}+G_{T}+G_{R}+10 \log \sigma-P_{R}-20 \log f\right. \\
& \left.-30 \log (4 \pi)+20 \log (c)-X_{G}\right]
\end{aligned}
$$

Let us consider $K_{1}=20 \log [4 \pi / c]$; so, ( 19) will yield the following relation,

$$
\begin{aligned}
10 \log R & \cong \frac{1}{4}\left[P_{T}+G_{T}+G_{R}+10 \log \sigma\right. \\
& \left.-P_{R}-X_{G}-20 \log f-K_{1}-10.99 d B\right]
\end{aligned}
$$

Finally, Wi-Fi radar range through log form can be computed as;

$$
\text { WiFi Radar Range }=R=10^{\frac{Q d B}{10}}
$$

\section{REFERENCES}

[1] Z. Zhou, C. Wu, Z. Yang, and Y. Liu, "Sensorless sensing with Wi-Fi," Tsinghua Science and Technology, vol. 20, no. 1, pp. 1-6, Feb. 2015.

[2] C. Phillips, D. Sicker, and D. Grunwald, "A survey of wireless path loss prediction and coverage mapping methods," IEEE Communications Surveys Tutorials, vol. 15, no. 1, pp. 255-270, First 2013.

[3] S. Y. Seidel and T. S. Rappaport, "914 MHz path loss prediction models for indoor wireless communications in multifloored buildings," IEEE Transactions on Antennas and Propagation, vol. 40, no. 2, pp. 207217, Feb 1992.

[4] N. Doulamis, P. Agrafiotis, G. Athanasiou, and A. Amditis, "Human object detection using very low resolution thermal cameras for urban search and rescue," in Proceedings of the 10th International Conference on PErvasive Technologies Related to Assistive Environments, ser. PETRA '17. New York, NY, USA: ACM, 2017, pp. 311-318.

[5] P. Agrafiotis, A. Doulamis, G. Athanasiou, and A. Amditis, "Real time earthquake's survivor detection using a miniaturized LWIR camera," in Proceedings of the 9th ACM International Conference on PErvasive Technologies Related to Assistive Environments - PETRA '16, ser. PETRA '16. ACM Press, 2016, pp. 21:1-21:4.

[6] H. Sun, P. Yang, L. Zu, and Q. Xu, "A far field sound source localization system for rescue robot," in Proceedings of IEEE International Conference on Control, Automation and Systems Engineering (CASE), Jul. 2011, pp. 1-4.

[7] J. Wang, Z. Cheng, L. Jing, and T. Yoshida, "Design of a 3D localization method for searching survivors after an earthquake based on WSN," in Proceedings of 3rd International Conference on Awareness Science and Technology (iCAST), Sept 2011, pp. 221-226.

[8] L. Crocco and V. Ferrara, "A review on ground penetrating radar technology for the detection of buried or trapped victims," in Proceedings of IEEE International Conference on Collaboration Technologies and Systems (CTS), May 2014, pp. 535-540.

[9] R. M. Narayanan, "Earthquake survivor detection using life signals from radar micro-doppler," in Proceedings of the 1st International Conference on Wireless Technologies for Humanitarian Relief, ser. ACWR '11. New York, NY, USA: ACM, 2011, pp. 259-264.

[10] Z. Li, H. Lv, Y. Zhang, G. Lu, S. Li, X. Jing, and J. Wang, "Detection of trapped survivors using 270/400 MHz dual-frequency IR-UWB radar based on time division multiplexing," in Proccedings of IEEE Topical Conference on Biomedical Wireless Technologies, Networks, and Sensing Systems (BioWireleSS), Jan 2014, pp. 31-33.

[11] F. JalaliBidgoli, S. Moghadami, and S. Ardalan, "A compact portable microwave life-detection device for finding survivors," IEEE Embedded Systems Letters, vol. 8, no. 1, pp. 10-13, March 2016.

[12] M. Li, Y. Meng, J. Liu, H. Zhu, X. Liang, Y. Liu, and N. Ruan, "When CSI meets public Wi-Fi," in Proceedings of the 2016 ACM SIGSAC Conference on Computer and Communications Security - CCS'16. ACM Press, 2016, pp. 1068-1079.

[13] H. Abdelnasser, M. Youssef, and K. A. Harras, "WiGest: A ubiquitous Wi-Fi-based gesture recognition system," in Proceedings of IEEE Conference on Computer Communications (INFOCOM), Apr. 2015, pp. 1472-1480.

[14] L. Gui, M. Yang, H. Yu, J. Li, F. Shu, and F. Xiao, "A cramer-rao lower bound of CSI-based indoor localization," IEEE Transactions on Vehicular Technology, vol. 67, no. 3, pp. 2814-2818, Mar. 2018.

[15] J. Zhang, B. Wei, W. Hu, and S. S. Kanhere, "WiFi-ID: Human identification using WiFi signal," in 2016 International Conference on Distributed Computing in Sensor Systems (DCOSS). IEEE, May 2016.

[16] G. Wang, Y. Zou, Z. Zhou, K. Wu, and L. M. Ni, "We can hear you with wi-fi!" in Proceedings of the 20th annual international conference on Mobile computing and networking - MobiCom '14. ACM Press, 2014.

[17] F. Li, C. Xu, Y. Liu, Y. Zhang, Z. Li, K. Sharif, and Y. Wang, "Mosleep: Unobtrusive sleep and movement monitoring via Wi-Fi signal," in Proceedings of IEEE 35th International Performance Computing and Communications Conference (IPCCC), Dec. 2016, pp. 1-8.

[18] X. Liu, J. Cao, S. Tang, J. Wen, and P. Guo, "Contactless respiration monitoring via off-the-shelf Wi-Fi devices," IEEE Transactions on Mobile Computing, vol. 15, no. 10, pp. 2466-2479, Oct. 2016.

[19] K. Ali, A. X. Liu, W. Wang, and M. Shahzad, "Keystroke recognition using Wi-Fi signals," in Proceedings of the 21st Annual International Conference on Mobile Computing and Networking - MobiCom '15. ACM Press, 2015, pp. 90-102.

[20] L. Sun, S. Sen, D. Koutsonikolas, and K.-H. Kim, "WiDraw," in Proceedings of the 21st Annual International Conference on Mobile Computing and Networking - MobiCom '15. ACM Press, 2015. 
[21] H. Wang, D. Zhang, Y. Wang, J. Ma, Y. Wang, and S. Li, "RT-Fall: A real-time and contactless fall detection system with commodity Wi-Fi devices," IEEE Transactions on Mobile Computing, vol. 16, no. 2, pp. 511-526, Feb. 2017.

[22] W. Wang, Y. Chen, and Q. Zhang, "Privacy-preserving location authentication in Wi-Fi networks using fine-grained physical layer signatures," IEEE Transactions on Wireless Communications, vol. 15, no. 2, pp. 1218-1225, Feb. 2016.

[23] A. D. Carlofelice, E. D. Giampaolo, M. Elaiopoulos, M. Feliziani, M. Roselli, and P. Tognolatti, "Localization of radio emitters into collapsed buildings after earthquake: Measurements of path loss and direction of arrival," in Proceedings of International Symposium on Electromagnetic Compatibility - EMC EUROPE, Sept 2012, pp. 1-6.

[24] A. DiCarlofelice, E. DiGiampaolo, M. Feliziani, and P. Tognolatti, "Experimental characterization of electromagnetic propagation under rubble of a historic town after disaster," IEEE Transactions on Vehicular Technology, vol. 64, no. 6, pp. 2288-2296, June 2015.

[25] C. L. Holloway, G. Koepke, D. Camell, W. F. Young, and K. A. Remley, "Propagation measurements before, during, and after the collapse of three large public buildings," IEEE Antennas and Propagation Magazine, vol. 56, no. 3, pp. 16-36, June 2014.

[26] A. Durantini and D. Cassioli, "A multi-wall path loss model for indoor UWB propagation," in Proceedings of IEEE 61st Vehicular Technology Conference, vol. 1, May 2005, pp. 30-34 Vol. 1.

[27] H. Okamoto, K. Kitao, and S. Ichitsubo, "Outdoor-to-indoor propagation loss prediction in $800-\mathrm{MHz}$ to $8-\mathrm{GHz}$ band for an urban area," IEEE Transactions on Vehicular Technology, vol. 58, no. 3, pp. 1059-1067, March 2009

[28] C. R. Anderson and T. S. Rappaport, "In-building wideband partition loss measurements at 2.5 and $60 \mathrm{GHz}$," IEEE Transactions on Wireless Communications, vol. 3, no. 3, pp. 922-928, May 2004

[29] A. Aragón-Zavala, Indoor Wireless Communications: From Theory to Implementation. Wiley, Sep. 2017. [Online]. Available: https: //onlinelibrary.wiley.com/doi/book/10.1002/9781119004547

[30] M. F. Khan, G. Wang, M. Z. A. Bhuiyan, and X. Li, "Wi-Fi signal coverage distance estimation in collapsed structures," in Proceedings of IEEE International Symposium on Parallel and Distributed Processing with Applications and IEEE International Conference on Ubiquitous Computing and Communications (ISPA/IUCC), Dec. 2017, pp. 10661073.

[31] M. F. Khan, G. Wang, and M. Z. A. Bhuiyan, "Wi-fi frequency selection concept for effective coverage in collapsed structures," Future Generation Computer Systems, vol. 97, pp. 409-424, Aug. 2019.

[32] M. F. Khan, G. Wang, M. Z. A. Bhuiyan, and T. Peng, "Wi-fi halow signal coverage estimation in collapsed structures," in 2018 IEEE 16th Intl Conf on Dependable, Autonomic and Secure Computing, 16th Intl Conf on Pervasive Intelligence and Computing, 4th Intl Conf on Big Data Intelligence and Computing and Cyber Science and Technology Congress(DASC/PiCom/DataCom/CyberSciTech). IEEE, Aug. 2018.

[33] M. F. Khan, G. Wang, M. Z. A. Bhuiyan, and X. Xing, "Towards wi-fi radar in collapsed structures," in 2018 IEEE SmartWorld, Ubiquitous Intelligence \& Computing, Advanced \& Trusted Computing, Scalable Computing \& Communications, Cloud \& Big Data Computing, Internet of People and Smart City Innovation (SmartWorld/SCALCOM/UIC/ATC/CBDCom/IOP/SCI). IEEE, Oct. 2018.

[34] S. Aust, R. V. Prasad, and I. G. M. M. Niemegeers, "Outdoor long-range wlans: A lesson for ieee 802.11ah," IEEE Communications Surveys Tutorials, vol. 17, no. 3, pp. 1761-1775, thirdquarter 2015.

[35] M. Z. A. Bhuiyan, G. Wang, J. Wu, J. Cao, X. Liu, and T. Wang, "Dependable structural health monitoring using wireless sensor networks," IEEE Transactions on Dependable and Secure Computing, vol. 14, no. 4 , pp. 363-376, July 2017.

[36] M. Z. A. Bhuiyan, J. Wu, G. Wang, Z. Chen, J. Chen, and T. Wang, "Quality-guaranteed event-sensitive data collection and monitoring in vibration sensor networks," IEEE Transactions on Industrial Informatics, vol. 13, no. 2, pp. 572-583, April 2017.

[37] M. Z. A. Bhuiyan, J. Wu, G. Wang, and J. Cao, "Sensing and decision making in cyber-physical systems: The case of structural event monitoring," IEEE Transactions on Industrial Informatics, vol. 12, no. 6, pp. 2103-2114, Dec 2016.

[38] V. Tiwari, P. K. Jain, and P. Tandon, "An integrated shannon entropy and TOPSIS for product design concept evaluation based on bijective soft set," Journal of Intelligent Manufacturing, Jul. 2017.

[39] S. Aust, R. V. Prasad, and I. G. M. M. Niemegeers, "Outdoor long-range WLANs: A lesson for IEEE 802.11ah," IEEE Communications Surveys \& Tutorials, vol. 17, no. 3, pp. 1761-1775, 2015.
[40] D. Molodtsov, "Soft set theory-first results," vol. 37, pp. 19-31, 02 1999.

[41] K. Gong, Z. Xiao, and X. Zhang, "The bijective soft set with its operations," Computers \& Mathematics with Applications, vol. 60, no. 8, pp. $2270-2278$, Oct. 2010

[42] A. J. C. R. C. B.-Y. Hayat Khizar, Ali Muhammad Irfan and T. K. U, "Best concept selection in design process: An application of generalized intuitionistic fuzzy soft sets," Journal of Intelligent \& Fuzzy Systems, vol. 35 , no. 5, pp. 5707-5720, Nov. 2018 .

[43] A. Roy and P. Maji, "A fuzzy soft set theoretic approach to decision making problems," Journal of Computational and Applied Mathematics, vol. 203, no. 2, pp. 412-418, Jun. 2007.

[44] T.-C. Wang and H.-D. Lee, "Developing a fuzzy TOPSIS approach based on subjective weights and objective weights," Expert Systems with Applications, vol. 36, no. 5, pp. 8980-8985, Jul. 2009.

[45] "IEEE standard for information technology - telecommunications and information exchange between systems - local and metropolitan area networks - specific requirements - part 11: Wireless LAN Medium Access Control (MAC) and Physical Layer (PHY) specifications," IEEE Std 802.11-2007 (Revision of IEEE Std 802.11-1999) - Redline, pp. 11238, June 2007

[46] V. Tiwari, P. K. Jain, and P. Tandon, "A bijective soft set theoretic approach for concept selection in design process," Journal of Engineering Design, vol. 28, no. 2, pp. 100-117, Jan. 2017. 